Article

\title{
Foot-Worn Inertial Sensors Are Reliable to Assess Spatiotemporal Gait Parameters in Axial Spondyloarthritis under Single and Dual Task Walking in Axial Spondyloarthritis
}

\author{
Julie Soulard ${ }^{1,2, *} \mathbb{C}^{\circ}$, Jacques Vaillant ${ }^{1}$, Romain Balaguier $\left.{ }^{1}{ }^{(}\right)$, Athan Baillet ${ }^{3}$, Philippe Gaudin ${ }^{3}$ \\ and Nicolas Vuillerme ${ }^{1,4,5}$ \\ 1 University Grenoble Alpes, AGEIS, 38000 Grenoble, France; jacques.vaillant@gmail.com (J.V.); \\ Romain.balaguier@hotmail.fr (R.B.); nicolas.vuillerme@univ-grenoble-alpes.fr (N.V.) \\ 2 CHU Grenoble Alpes, 38000 Grenoble, France \\ 3 University Grenoble Alpes, CNRS, CHU Grenoble Alpes, Grenoble INP, TIMC-IMAG UMR5525, \\ 38000 Grenoble, France; abaillet@chu-grenoble.fr (A.B.); pgaudin@chu-grenoble.fr (P.G.) \\ 4 Institut Universitaire de France, 75000 Paris, France \\ 5 LabCom Telecom4Health, University Grenoble Alpes \& Orange Labs, 38000 Grenoble, France \\ * Correspondence: jsoulard@chu-grenoble.fr
}

Received: 7 October 2020; Accepted: 9 November 2020; Published: 12 November 2020

check for updates

\begin{abstract}
The aim of this study was (1) to evaluate the relative and absolute reliability of gait parameters during walking in single- and dual-task conditions in patients with axial spondyloarthritis (axSpA), (2) to evaluate the absolute and relative reliability of dual task effects (DTE) parameters, and (3) to determine the number of trials required to ensure reliable gait assessment, in patients with axSpA. Twenty patients with axSpa performed a 10-m walk test in single- and dual-task conditions, three times for each condition. Spatiotemporal, symmetry, and DTE gait parameters were calculated from foot-worn inertial sensors. The relative reliability (intraclass correlation coefficients-ICC) and absolute reliability (standard error of measurement-SEM and minimum detectable change-MDC) were calculated for these parameters in each condition. Spatiotemporal gait parameters showed good to excellent reliability in both conditions $(0.59<$ ICC $<0.90)$. The reliability of symmetry and DTE parameters was low. ICC, SEM, and MDC were better when using the mean of the second and the third trials. Spatiotemporal gait parameters obtained from foot-worn inertial sensors assessed in patients with axSpA in single- and dual-task conditions are reliable. However, symmetry and DTE parameters seem less reliable and need to be interpreted with caution. Finally, better reliability of gait parameters was found when using the mean of the 2nd and the 3rd trials.
\end{abstract}

Keywords: ankylosing spondylitis; spondylarthritis; walking; wearable sensors; 10-m walk test; reliability; manual task; dual-task

\section{Introduction}

Gait is a fundamental human motor activity [1] and allows for movement from place to place in daily life, and thus social participation [2]. Assessing gait does offer undeniable prospects in a clinical setting. For instance, slow gait speed (i.e., $<1.0 \mathrm{~m} / \mathrm{s}$ ) is indeed associated to three times greater limitations in social participation [2]. Furthermore, gait outcomes can be used to quantify therapeutic interventions [3], or predict diverse outcomes including falls [4], cognitive decline [5], or mortality [6].

To assess gait performance, wearable inertial sensors are now affordable, transportable, and easy to use [7]. These devices advantageously allow for the computation of spatiotemporal gait parameters 
and could help to better evaluate gait characteristics in clinical practice [7]. Wearable inertial sensors can be placed, for instance, on the trunk, shank, or feet to perform gait assessment in a corridor, using devices easily transportable between care units. Wearable sensors allow for the analysis of multiple gait cycles in longer distances than laboratory-based gait assessments, which could even be performed continuously in a patients' home in an ecological environment. Moreover, to better adapt gait test to the motor-cognitive challenges that occur during every-day ambulation, dual-tasking paradigms are widely used in the clinical setting [8]. This consists of "the concurrent performance of two tasks that can be performed independently and have distinct and separate goals" [9] and was considered as closer to daily-living walking [10]. The use of gait dual-tasking paradigm has been shown to allow better classification and diagnosis in various populations such as patients with neurologic disorders (i.e., Parkinson disease [11], multiple sclerosis [12] or stroke [13]), with knee pain [14] or osteoarthritis [15], or older adults with cognitive impairment [16] or at risk of falls [17,18]. Dual task effects (DTE) assess the change in performance on the primary task (e.g., gait) while concurrently performing a secondary task (e.g., counting backward, carrying a full cup of water ... ) [19].

To separate disease or treatments effects to measurement errors of the system (i.e., inertial sensors), the reliability of these devices has to be evaluated [20]. More precisely, the intra-session reliability refers to the reliability of different trials performed during the same session [21,22]. It is recommended to better describe the sources and magnitudes of errors in gait analysis [21,23] and to compare performances between groups at a single time point [24]. Intra-session reliability can favorably be used to assess the number of trials to ensure reliable measurements [25-28] to further optimize gait assessments in clinical practice. The relative reliability (i.e., intraclass correlation coefficients (ICC)) and the absolute reliability (i.e., standard error of measurement (SEM) and minimum detectable change (MDC)) have complementary to be reported and are crucial in rehabilitation to interpret changing scores in patients [29]. Axial spondyloarthritis (axSpA) is a chronic rheumatic disease, which affects the vertebral column and sacroiliac joints [30]. This disease can lead to postural changes such as loss of lumbar lordosis, exaggeration of thoracic kyphosis, and difficulty in looking upwards [31]. Static and dynamic postural changes [32-34] and foot abnormalities [35] can also be associated, and thus have consequences on spatiotemporal and kinematic gait parameters, i.e., gait performance [36]. Previous studies [37-40] reported decreased lower limb angles in the sagittal plane and decreased pelvic tilt associated to lower variability among patients with axSpA compared to healthy controls [41]. However, to the best of our knowledge, no study has assessed the reliability of gait assessments in this population.

Thus, the aim of this study was to evaluate the intra-session reliability of gait assessment with foot-worn wearable inertial sensors, in single- and dual-task conditions, in patients with axSpA. More precisely, the aims were (1) to evaluate the relative and absolute reliability of gait parameters during walking in single- and dual-task conditions in patients with axSpA, (2) to evaluate the absolute and relative reliability of DTE parameters, and (3) to determine the number of trials required to ensure reliable gait assessment, in patients with axSpA.

\section{Materials and Methods}

\subsection{Participants}

Inclusion criteria were previously described [42]: (i) age between 18 to 65 years, (ii) diagnosis of axSpA according to ASAS/EULAR criteria [43] or ankylosing spondylitis according to Van der Linden et al. [44], (iii) ability to walk $180 \mathrm{~m}$ without technical help, and (iv) stable treatment for at least 12 months.

All participants signed informed consent and approved to participate in the FOLOMI study (Function, Locomotion, Measurement and Inflammation), a prospective longitudinal study [42]. The study was approved by local ethic committee (CPP Ile De France 1, RCB: 2017-A03468-45, date of agreement: 17 July 2018, Last version: V6.0, 17 June 2020) and is registered in Clinical trials (NCT03761212). The present study respects the GRRAS guidelines regarding psychometric studies [45]. 
The sample size was calculated using sample size calculator [46,47] with a minimum acceptable reliability of 0.6 , expected reliability of 0.85 , significance level of 0.05 , power of $80 \%$, and 3 repetitions per subject, giving a sample size of 18 .

\subsection{Experimental Protocol}

Patients' characteristics (age, weight, height, body mass index) and disease characteristics (disease duration, pain intensity at time of evaluation assessed with visual analogue scale, BASDAI, BASFI, and morning stiffness) were collected.

Gait was assessed at least 2 hours from the end of morning stiffness of the patients as morning stiffness can have consequences on functional limitations [48]. A 10-meter walk test (10 MWT) was performed on a 14-m walkway, at comfortable walking speed [49], in single- and dual-task conditions ( 3 trials per condition). In the dual-task condition, participants had to carry a full cup of water in their dominant hand with the following instruction: "perform both tasks as well as possible" [50,51]. The examiner noted whether there was any spillage of water [51]. Gait assessments were performed by the same examiner (JS). Participants wore walking shoes, with 2 inertial measurement units with tri-axial accelerometers and gyroscopes (Physilog $5^{\circledR}, 200 \mathrm{~Hz}$, BioAGM, Gait Up, CH), placed above both feet (behind the base of the fifth metatarsal) [52].

\subsection{Spatiotemporal Gait Parameters}

Spatiotemporal gait parameters were calculated with the Gait Analysis Software (V5.3.0) provided by Gait Up (CH) [53]. The two first and last steps were removed from the analysis [54,55] and at least 16 steps were included in the analysis [56]. After checking for non-significant differences between spatiotemporal gait parameters of left and right feet, the mean of left and right feet for each parameter was calculated:

- $\quad$ Speed $\left(\mathrm{m} . \mathrm{s}^{-1}\right)$ : Mean walking stride velocity of forward walking.

- Cadence (step/minute): Number of steps in a minute.

- Stride length (m): Distance between two consecutive footprints on the ground, from the heel of a foot to the heel of the same foot, one cycle after.

- Swing (\%): Portion of the cycle during which the foot is in the air and does not touch the ground.

- Stance (\%): Portion of the cycle during which part of the foot touches the ground.

- Double support (\%): Portion of the cycle where both feet touch the ground.

- Load (\%): Portion of the stance between the heel strike and the foot being flat on the ground.

- Foot Flat (\%): Portion of the stance where the foot is fully flat on the ground.

- Push (\%): Portion of the stance between the foot being flat on the ground and the toe leaving the ground at take-off.

In order to remove the variability between subject associated with size [57], speed, cadence, and stride length were normalized to have non dimensional values using the formula proposed by Hof [58] and Pinzone et al. [57]:

$$
\text { Normalized speed }(\text { speed } N)=\frac{\text { Speed }}{\sqrt{g l_{0}}}
$$

where $g$ denotes the acceleration of gravity $\left(=9.81 \mathrm{~m} / \mathrm{s}^{2}\right)$ and $l_{0}$ leg length.

$$
\text { Normalized cadence }(\text { cadence } N)=\text { Cadence } * \sqrt{\frac{l_{0}}{g}}
$$


where $g$ is the acceleration of gravity and $l_{0}$ leg length.

$$
\text { Normalized stride length }(\text { stride length } N)=\frac{\text { stride length }}{l_{0}}
$$

where $l_{0}$ denotes leg length.

\subsection{Symmetry Gait Parameters}

The symmetry index (SI) is the most commonly used and cited parameters [59]. It assesses symmetry between left and right feet, with a value of $0 \%$ indicating full symmetry and a value of $100 \%$ indicating asymmetry. SI was calculated with the following formula for each of the spatiotemporal gait parameter (except double support) [59]:

$$
\text { SymmetryIndex }(S I)=\frac{\left|X_{L}-X_{R}\right|}{0.5 \times\left(X_{L}+X_{R}\right)} \times 100 \%
$$

where $X_{L}$ is the value of each parameter of left foot and $X_{R}$ the value of each parameter of right foot.

The symmetry ratio (SR), described as easier to interpret and recommended on the basis of potential clinical utility in stroke patients [60] was also computed. A $S R$ of 1 indicates full symmetry, while a $S R$ of 2.0 on gait speed indicates that the right foot is twice faster than left foot. It was calculated with the following formula for each of the spatiotemporal gait parameter (except double support) [60]:

$$
\text { Symmetry ratio }(S R)=\frac{X_{R}}{X_{L}}
$$

where $X_{L}$ denotes the value of each spatiotemporal gait parameter of left foot and $X_{R}$ the value of each spatiotemporal gait parameter of right foot.

\subsection{Dual Task Effects Parameters}

To assess the influence of the addition of a secondary attention demanding task on gait, the dual task effect (DTE) was also calculated [9,51]. DTE is the relative measure of change in performance [61]. DTE calculated while walking and carrying a cup has previously been shown to be more reliable than when calculated while walking and performing a cognitive task [62]. DTE was calculated from mean parameters as proposed by [51]:

$$
D T E=\text { single task performance }- \text { dual task performance }
$$

DTE has the same unit as the gait parameter that is used in the calculation. A negative value of $D T E$ for gait speed, cadence, stride length, swing, and foot flat is associated to better performance in the dual-task, while a negative value for stance, double support, load, and push is associated to worse performance in the dual-task [51].

DTE can also be calculated as a percentage, being unit-less, and permitting comparison between parameters [51]:

$$
D T E \%=\frac{\text { single task performance }- \text { dual task performance }}{\text { single task performance }} \times 100
$$

The same interpretation as DTE can be done regarding positive and negative DTE $\%$ values.

\subsection{Data Analysis}

Spatiotemporal gait parameters and symmetry gait parameters in single- and dual-task conditions, and DTE and DTE\% values were normally distributed (Shapiro-Wilk normality test). 
To estimate the magnitude of the systematic difference in gait parameters between trials 1-2, trials 1-3, trials 2-3, 95\% confidence interval (CI) of the mean difference $\left(\right.$ Mean $\left._{\text {Diff }}\right)$ were used. CI was calculated with the following formula:

$$
C I=\text { Mean }_{\text {Diff }} \pm \operatorname{tn}-1 \times \sqrt{ }\left(\frac{S D_{\text {Diff }}}{n}\right)
$$

where $n$ corresponds to the number of participants and $t n-1$ corresponds to the value of $t$ distribution with $n-1$ of freedom.

A repeated measures analysis of variance (RM-ANOVA) with the number of trials used as within subject factor was performed on gait parameters, ICC and SEM values. When significant effect of the number of trials was found, a Tukey post-hoc test was used to compare differences between trials. ICC, SEM, and MDC were used to compute the relative and absolute reliability across the trials 1-2-3 in each condition (single- and dual-task). The relative reliability was evaluated by the calculation of a 2-way fixed $\mathrm{ICC}_{2,1}$ (for absolute agreement). ICC values inferior to 0 were considered as poor reliability, between 0.01 to 0.2 as slight, between $0.21-0.4$ as fair, between $0.41-0.6$ as moderate, $0.61-0.8$ as substantial, and $0.81-1.00$ as almost perfect [63]. SEM, with the same unit as gait parameters, corresponds to the absolute measure of the variability of the errors of measurements and informs on the precision of gait parameters of individual participants [64]. SEM was calculated with the following formula [65]:

$$
S E M=S D \sqrt{ }(1-I C C)
$$

where $S D$ is the standard deviation of the parameters from all patients, and ICC the relative reliability. $M D C$ is the minimum value for which a difference can be considered as "real", and was generated with the formula:

$$
M D C=S E M \times 1.96 \times \sqrt{ } 2
$$

$S E M \%$ and $M D C \%$ were also calculated as a percentage of mean for each parameter.

Bland and Altman plots of the differences between trials against their mean and limits of agreements (LOA) were used to assess the magnitude of disagreement between trials. A difference between trials outside the LOA can be considered as real change.

ICC, SEM (3), MDC (4), SEM\%, and MDC\% were calculated with the same formula for DTE (1) and DTE\% (2) [66].

\section{Results}

Twenty patients with axSpA (10 first men and 10 first women included in FOLOMI study) were included in the present study. Patients were 44.9 (10.7) years old, 169.0 (8.7) cm height, 74.9 (15.1) body weight, with a disease duration of 11.2 (9.5) years. Pain intensity at the time of evaluation was 3.3 (2.5) and morning stiffness was about 32.5 (32.3) minutes. BASDAI was 3.3 (1.9) and BASFI was 3.1 (2.0).

\subsection{Intra-Session Relative and Absolute Reliability of Spatiotemporal Gait Parameters}

Tables 1 and 2 show, respectively, the means and standard deviations of spatiotemporal gait parameters obtained in the single- and the dual-task conditions for each trial, the mean of the three trials, and $p$-values obtained from RM-ANOVA with differences between trials.

Tables 3 and 4 show relative (ICC) and absolute (SEM\% and MDC\%) reliability of spatiotemporal gait parameters for each trial, respectively, in single and dual-task conditions. 
Table 1. Mean and standard deviation (SD) of spatiotemporal, normalized (N) and symmetry (symmetry index-SI and symmetry ratio-SR) gait parameters obtained in the single-task condition, and ANOVA results.

\begin{tabular}{|c|c|c|c|c|c|c|c|c|c|c|c|}
\hline & \multicolumn{2}{|c|}{ Trial 1} & \multicolumn{2}{|c|}{ Trial 2} & \multicolumn{2}{|c|}{ Trial 3} & \multicolumn{2}{|c|}{ Trial 1-2-3 } & \multirow{2}{*}{$\begin{array}{c}\text { T1-T2 } \\
p \text {-Value }\end{array}$} & \multirow{2}{*}{$\begin{array}{c}\text { T1-T3 } \\
p \text {-Value }\end{array}$} & \multirow{2}{*}{$\begin{array}{c}\text { T2-T3 } \\
p \text {-Value }\end{array}$} \\
\hline & Mean & SD & Mean & SD & Mean & SD & Mean & SD & & & \\
\hline \multicolumn{12}{|c|}{ Spatiotemporal parameters } \\
\hline Speed & 1.24 & 0.20 & 1.26 & 0.20 & 1.27 & 0.19 & 1.26 & 0.19 & 0.95 & 0.86 & 0.97 \\
\hline Cadence & 107.25 & 9.47 & 108.20 & 9.16 & 108.83 & 8.70 & 108.09 & 8.98 & 0.94 & 0.85 & 0.97 \\
\hline Stride length & 1.37 & 0.17 & 1.38 & 0.17 & 1.38 & 0.17 & 1.38 & 0.17 & 0.97 & 0.95 & 1.00 \\
\hline Swing & 38.03 & 1.69 & 37.96 & 1.29 & 38.15 & 1.54 & 38.05 & 1.49 & 0.99 & 0.97 & 0.92 \\
\hline Stance & 61.97 & 1.69 & 62.04 & 1.29 & 61.85 & 1.54 & 61.95 & 1.49 & 0.99 & 0.97 & 0.92 \\
\hline Double support & 23.49 & 3.51 & 23.62 & 2.36 & 23.35 & 2.54 & 23.49 & 2.81 & 0.99 & 0.99 & 0.95 \\
\hline Load & 12.71 & 3.15 & 12.69 & 3.06 & 13.03 & 3.25 & 12.81 & 3.10 & 1.00 & 0.94 & 0.94 \\
\hline Foot flat & 52.54 & 6.00 & 52.31 & 4.79 & 52.04 & 5.08 & 52.30 & 5.23 & 0.99 & 0.95 & 0.99 \\
\hline Push & 34.75 & 4.00 & 35.00 & 2.79 & 34.94 & 3.39 & 34.90 & 3.37 & 0.97 & 0.98 & 1.00 \\
\hline \multicolumn{12}{|c|}{ Normalized parameters } \\
\hline Speed N & 0.42 & 0.06 & 0.42 & 0.06 & 0.43 & 0.06 & 0.42 & 0.06 & 0.95 & 0.85 & 0.97 \\
\hline Cadence N & 32.38 & 2.77 & 32.58 & 2.73 & 32.77 & 2.56 & 32.58 & 2.65 & 0.97 & 0.89 & 0.97 \\
\hline Stride length $N$ & 1.52 & 0.16 & 1.54 & 0.16 & 1.54 & 0.16 & 1.54 & 0.16 & 0.96 & 0.93 & 0.99 \\
\hline \multicolumn{12}{|c|}{ Symmetry index parameters } \\
\hline Speed SI & 1.60 & 1.13 & 2.38 & 2.81 & 2.20 & 2.00 & 2.06 & 2.09 & 0.47 & 0.63 & 0.96 \\
\hline Cadence SI & 1.01 & 1.13 & 1.50 & 3.03 & 1.62 & 2.97 & 1.38 & 2.51 & 0.82 & 0.73 & 0.99 \\
\hline Stride length SI & 1.46 & 1.08 & 1.79 & 1.48 & 1.88 & 1.76 & 1.71 & 1.45 & 0.76 & 0.64 & 0.98 \\
\hline Swing SI & 3.08 & 2.07 & 2.98 & 2.21 & 3.28 & 3.64 & 3.11 & 2.69 & 0.99 & 0.97 & 0.94 \\
\hline Stance SI & 1.87 & 1.23 & 1.80 & 1.27 & 1.96 & 2.06 & 1.88 & 1.54 & 0.99 & 0.98 & 0.95 \\
\hline Load SI & 10.45 & 10.12 & 13.72 & 9.69 & 13.08 & 8.64 & 12.42 & 9.45 & 0.53 & 0.66 & 0.98 \\
\hline Foot flat SI & 3.66 & 2.55 & 5.24 & 4.25 & 4.83 & 4.64 & 4.58 & 3.91 & 0.42 & 0.62 & 0.94 \\
\hline Push SI & 6.11 & 4.86 & 6.42 & 5.70 & 5.93 & 5.75 & 6.15 & 5.36 & 0.98 & 0.99 & 0.96 \\
\hline \multicolumn{12}{|c|}{ Symmetry ratio parameters } \\
\hline Speed SR & 1.01 & 0.02 & 1.01 & 0.04 & 1.02 & 0.02 & 1.01 & 0.03 & 1.00 & 0.37 & 0.36 \\
\hline Cadence SR & 1.00 & 0.01 & 1.00 & 0.03 & 1.01 & 0.03 & 1.00 & 0.03 & 0.94 & 0.36 & 0.56 \\
\hline Stride length SR & 1.01 & 0.02 & 1.00 & 0.02 & 1.02 & 0.02 & 1.01 & 0.02 & 0.67 & 0.59 & 0.17 \\
\hline Swing SR & 1.00 & 0.04 & 1.00 & 0.04 & 1.02 & 0.05 & 1.00 & 0.04 & 1.00 & 0.41 & 0.40 \\
\hline Stance SR & 1.00 & 0.02 & 1.00 & 0.02 & 0.99 & 0.03 & 1.00 & 0.02 & 0.99 & 0.47 & 0.41 \\
\hline Load SR & 1.01 & 0.15 & 1.01 & 0.17 & 1.03 & 0.15 & 1.02 & 0.16 & 1.00 & 0.97 & 0.96 \\
\hline Foot flat SR & 0.99 & 0.04 & 0.99 & 0.06 & 0.98 & 0.06 & 0.98 & 0.06 & 0.99 & 0.83 & 0.90 \\
\hline Push SR & 1.02 & 0.08 & 1.02 & 0.09 & 1.03 & 0.08 & 1.02 & 0.08 & 1.00 & 0.92 & 0.96 \\
\hline
\end{tabular}


Table 2. Mean and standard deviation (SD) of spatiotemporal, normalized (N) and symmetry (symmetry index-SI and symmetry ratio-SR) gait parameters obtained in the dual-task condition, and ANOVA results.

\begin{tabular}{|c|c|c|c|c|c|c|c|c|c|c|c|}
\hline & \multicolumn{2}{|c|}{ Trial 1} & \multicolumn{2}{|c|}{ Trial 2} & \multicolumn{2}{|c|}{ Trial 3} & \multicolumn{2}{|c|}{ Trial 1-2-3 } & \multirow{2}{*}{$\begin{array}{c}\text { T1-T2 } \\
p \text {-Value }\end{array}$} & \multirow{2}{*}{$\begin{array}{c}\text { T1-T3 } \\
p \text {-Value }\end{array}$} & \multirow{2}{*}{$\begin{array}{c}\mathrm{T} 2-\mathrm{T} 3 \\
p \text {-Value }\end{array}$} \\
\hline & Mean & SD & Mean & SD & Mean & SD & Mean & SD & & & \\
\hline \multicolumn{12}{|c|}{ Spatiotemporal parameters } \\
\hline Speed & 1.17 & 0.21 & 1.18 & 0.26 & 1.17 & 0.23 & 1.18 & 0.23 & 0.99 & 1.00 & 0.98 \\
\hline Cadence & 106.91 & 9.45 & 106.64 & 10.80 & 106.56 & 11.62 & 106.71 & 10.45 & 1.00 & 0.99 & 1.00 \\
\hline Stride length & 1.30 & 0.18 & 1.30 & 0.21 & 1.29 & 0.17 & 1.30 & 0.19 & 1.00 & 1.00 & 0.99 \\
\hline Swing & 37.47 & 1.33 & 37.48 & 1.92 & 37.42 & 1.46 & 37.46 & 1.56 & 1.00 & 0.99 & 0.99 \\
\hline Stance & 62.53 & 1.33 & 62.52 & 1.92 & 62.58 & 1.46 & 62.54 & 1.56 & 1.00 & 0.99 & 0.99 \\
\hline Double support & 24.81 & 2.47 & 24.13 & 3.27 & 24.86 & 3.07 & 24.61 & 2.91 & 0.76 & 1.00 & 0.73 \\
\hline Load & 12.79 & 3.67 & 12.83 & 3.74 & 12.67 & 3.40 & 12.76 & 3.54 & 1.00 & 0.99 & 0.99 \\
\hline Foot flat & 52.31 & 5.36 & 52.42 & 6.07 & 52.73 & 5.85 & 52.48 & 5.66 & 1.00 & 0.97 & 0.99 \\
\hline Push & 34.90 & 3.52 & 34.76 & 4.08 & 34.60 & 4.21 & 34.75 & 3.87 & 0.99 & 0.97 & 0.99 \\
\hline \multicolumn{12}{|c|}{ Normalized parameters } \\
\hline Speed N & 0.40 & 0.07 & 0.40 & 0.08 & 0.39 & 0.07 & 0.40 & 0.07 & 0.99 & 1.00 & 0.98 \\
\hline Cadence N & 33.00 & 2.92 & 32.92 & 3.33 & 32.90 & 3.59 & 32.94 & 3.23 & 1.00 & 0.99 & 1.00 \\
\hline Stride length $\mathrm{N}$ & 1.45 & 0.17 & 1.45 & 0.20 & 1.45 & 0.17 & 1.45 & 0.18 & 1.00 & 1.00 & 0.99 \\
\hline \multicolumn{12}{|c|}{ Symmetry index parameters } \\
\hline Speed SI & 1.55 & 0.96 & 1.89 & 2.16 & 1.30 & 1.27 & 1.58 & 1.53 & 0.78 & 0.87 & 0.48 \\
\hline Cadence SI & 0.78 & 0.45 & 1.47 & 2.08 & 0.65 & 0.32 & 0.96 & 1.27 & 0.20 & 0.94 & 0.11 \\
\hline Stride length SI & 1.28 & 0.79 & 1.40 & 1.02 & 1.33 & 1.22 & 1.33 & 1.00 & 0.93 & 0.99 & 0.98 \\
\hline Swing SI & 2.87 & 2.66 & 2.38 & 1.83 & 4.01 & 3.72 & 3.09 & 2.87 & 0.85 & 0.43 & 0.19 \\
\hline Stance SI & 1.72 & 1.55 & 1.44 & 1.13 & 2.37 & 2.16 & 1.84 & 1.68 & 0.87 & 0.44 & 0.21 \\
\hline Load SI & 11.22 & 8.54 & 9.44 & 9.09 & 15.05 & 12.21 & 11.89 & 10.15 & 0.84 & 0.46 & 0.21 \\
\hline Foot flat SI & 5.04 & 4.78 & 5.21 & 3.54 & 5.64 & 3.84 & 5.29 & 4.04 & 0.99 & 0.89 & 0.95 \\
\hline Push SI & 5.53 & 4.96 & 6.59 & 5.11 & 5.94 & 4.09 & 6.01 & 4.68 & 0.76 & 0.96 & 0.91 \\
\hline \multicolumn{12}{|c|}{ Symmetry ratio parameters } \\
\hline Speed SR & 1.01 & 0.01 & 1.01 & 0.03 & 1.01 & 0.02 & 1.01 & 0.02 & 0.96 & 0.90 & 0.98 \\
\hline Cadence SR & 1.00 & 0.01 & 1.00 & 0.03 & 1.00 & 0.01 & 1.00 & 0.02 & 0.96 & 0.96 & 0.85 \\
\hline Stride length SR & 1.01 & 0.01 & 1.01 & 0.01 & 1.01 & 0.02 & 1.01 & 0.01 & 0.88 & 0.89 & 0.63 \\
\hline Swing SR & 0.99 & 0.04 & 0.99 & 0.03 & 1.00 & 0.06 & 0.99 & 0.04 & 0.98 & 0.58 & 0.69 \\
\hline Stance SR & 1.01 & 0.02 & 1.01 & 0.02 & 1.00 & 0.03 & 1.01 & 0.02 & 0.98 & 0.61 & 0.73 \\
\hline Load SR & 1.04 & 0.15 & 1.01 & 0.12 & 1.03 & 0.18 & 1.03 & 0.15 & 0.87 & 1.00 & 0.92 \\
\hline Foot flat SR & 0.97 & 0.06 & 0.98 & 0.06 & 0.97 & 0.06 & 0.97 & 0.06 & 0.99 & 1.00 & 0.98 \\
\hline Push SR & 1.02 & 0.07 & 1.03 & 0.08 & 1.03 & 0.07 & 1.03 & 0.07 & 1.00 & 1.00 & 1.00 \\
\hline
\end{tabular}


Table 3. ICC, SEM and MDC values for spatiotemporal, normalized (N) and symmetry (symmetry index-SI and symmetry ratio-SR) gait parameters obtained in the single-task condition between the mean of the first and the second trials (T1-T2), the first and the third trials (T1-T3), the second and the third trials (T2-T3) and the means of the three consecutive trials (T1-T2-T3).

\begin{tabular}{|c|c|c|c|c|c|c|c|c|c|c|c|c|c|}
\hline & \multirow[b]{2}{*}{ Parameters } & \multicolumn{3}{|c|}{ T1-T2 } & \multicolumn{3}{|c|}{ T1-T3 } & \multicolumn{3}{|c|}{ T2-T3 } & \multicolumn{3}{|c|}{ T1-2-3 } \\
\hline & & ICC (lb-ub) & SEM(\%) & MDC(\%) & ICC (lb-ub) & SEM(\%) & MDC(\%) & ICC (lb-ub) & SEM(\%) & MDC(\%) & ICC (lb-ub) & SEM(\%) & MDC(\%) \\
\hline \multirow{8}{*}{$\begin{array}{l}\text { Almost perfect } \\
0.81<\text { ICC }<1\end{array}$} & \multicolumn{13}{|c|}{ Spatiotemporal parameters } \\
\hline & speed & $0.96(0.92-0.98)$ & 2.99 & 8.29 & $0.96(0.87-0.98)$ & 3.24 & 8.99 & $0.98(0.95-0.99)$ & 2.33 & 6.46 & $0.97(0.93-0.98)$ & 2.87 & 7.95 \\
\hline & cadence & $0.95(0.90-0.98)$ & 1.90 & 5.28 & $0.95(0.88-0.98)$ & 1.89 & 5.25 & $0.97(0.94-0.99)$ & 1.35 & 3.75 & $0.96(0.92-0.98)$ & 1.73 & 4.79 \\
\hline & stride length & $0.98(0.95-0.99)$ & 1.82 & 5.04 & $0.97(0.94-0.99)$ & 2.02 & 5.60 & $0.98(0.97-0.99)$ & 1.50 & 4.15 & $0.98(0.96-0.99)$ & 1.78 & 4.94 \\
\hline & & $0.81(0.64-0.91)$ & 5.41 & 14.99 & $0.59(0.29-0.79)$ & 8.27 & 22.91 & $0.82(0.64-0.91)$ & 4.43 & 12.27 & $0.74(0.58-0.86)$ & 6.14 & 17.01 \\
\hline & swing & $0.74(0.52-0.87)$ & 1.98 & 5.49 & $0.68(0.43-0.84)$ & 2.36 & 6.53 & $0.85(0.70-0.93)$ & 1.45 & 4.01 & $0.75(0.60-0.87)$ & 1.96 & 5.43 \\
\hline & stance & $0.74(0.52-0.87)$ & 1.21 & 3.36 & $0.68(0.43-0.84)$ & 1.45 & 4.02 & $0.85(0.70-0.93)$ & 0.89 & 2.46 & $0.75(0.60-0.87)$ & 1.20 & 3.33 \\
\hline & LDr & $0.93(0.85-0.97)$ & 6.54 & 18.13 & $0.94(0.87-0.97)$ & 6.24 & 17.29 & $0.95(0.88-0.97)$ & 5.68 & 15.75 & $0.94(0.89-0.97)$ & 6.14 & 17.01 \\
\hline \multirow{6}{*}{$\begin{array}{c}\text { Substantial } \\
0.61<\text { ICC }<0.8\end{array}$} & FFr & $0.90(0.80-0.95)$ & 3.20 & 8.87 & $0.93(0.85-0.97)$ & 2.83 & 7.84 & $0.97(0.93-0.98)$ & 1.75 & 4.85 & $0.93(0.87-0.96)$ & 2.66 & 7.36 \\
\hline & Pur & $0.86(0.72-0.93)$ & 3.69 & 10.24 & $0.88(0.76-0.94)$ & 3.68 & 10.19 & $0.93(0.86-0.97)$ & 2.30 & 6.37 & $0.89(0.80-0.94)$ & 3.27 & 9.08 \\
\hline & \multicolumn{13}{|c|}{ Normalized parameters } \\
\hline & speed $\mathrm{N}$ & $0.96(0.92-0.98)$ & 2.98 & 8.25 & $0.95(0.86-0.98)$ & 3.21 & 8.90 & $0.97(0.95-0.99)$ & 2.32 & 6.44 & $0.96(0.93-0.98)$ & 2.85 & 7.90 \\
\hline & cadence $\mathrm{N}$ & $0.95(0.89-0.98)$ & 1.90 & 5.27 & $0.94(0.87-0.97)$ & 1.90 & 5.28 & $0.97(0.94-0.99)$ & 1.35 & 3.75 & $0.95(0.92-0.98)$ & 1.73 & 4.80 \\
\hline & slength $\mathrm{N}$ & $0.97(0.93-0.99)$ & 1.81 & 5.02 & $0.96(0.91-0.98)$ & 1.99 & 5.52 & $0.98(0.95-0.99)$ & 1.50 & 4.15 & $0.97(0.94-0.98)$ & 1.77 & 4.91 \\
\hline \multirow{6}{*}{$\begin{array}{c}\text { Moderate } \\
0.41<\text { ICC }<0.6\end{array}$} & \multicolumn{13}{|c|}{ Symmetry index parameters } \\
\hline & speed SI & $0.03(-0.34-0.39)$ & 106.65 & 295.61 & $0.08(-0.28-0.43)$ & 82.51 & 228.70 & $0.66(0.39-0.83)$ & 61.08 & 169.30 & $0.32(0.09-0.56)$ & 83.67 & 231.93 \\
\hline & cadence SI & $0.00(-0.37-0.37)$ & 181.24 & 502.38 & $0.00(-0.37-0.37)$ & 170.39 & 472.31 & $0.68(0.42-0.84)$ & 107.73 & 298.61 & $0.26(0.03-0.51)$ & 156.66 & 434.25 \\
\hline & slength SI & $0.00(-0.37-0.37)$ & 79.43 & 220.16 & $0.00(-0.37-0.37)$ & 87.28 & 241.93 & $\begin{array}{c}0.25 \\
(-0.12-0.57)\end{array}$ & 75.81 & 210.12 & $0.04(-0.15-0.3)$ & 83.19 & 230.60 \\
\hline & swing SI & $0.00(-0.37-0.37)$ & 69.83 & 193.55 & $0.15(-0.23-0.49)$ & 84.89 & 235.32 & $0.53(0.21-0.75)$ & 65.12 & 180.51 & $0.26(0.03-0.51)$ & 74.56 & 206.66 \\
\hline & stance SI & $0.00(-0.37-0.37)$ & 67.17 & 186.18 & $0.13(-0.25-0.48)$ & 81.53 & 225.99 & $0.48(0.15-0.72)$ & 64.66 & 179.22 & $0.23(0.01-0.49)$ & 71.99 & 199.54 \\
\hline \multirow{6}{*}{$\begin{array}{c}\quad \text { Fair } \\
0.21<\text { ICC }<0.4\end{array}$} & LDr SI & $0.33(-0.03-0.62)$ & 67.07 & 185.92 & $0.56(0.25-0.77)$ & 52.90 & 146.63 & $\begin{array}{c}0.30 \\
(-0.07-0.60)\end{array}$ & 56.45 & 156.47 & $0.40(0.17-0.63)$ & 59.16 & 163.99 \\
\hline & FFr SI & $0.53(0.21-0.75)$ & 54.81 & 151.92 & $0.55(0.24-0.76)$ & 59.34 & 164.48 & $0.75(0.53-0.88)$ & 43.68 & 121.08 & $0.63(0.43-0.79)$ & 52.31 & 144.99 \\
\hline & Pur SI & $0.40(0.05-0.67)$ & 64.37 & 178.43 & $0.42(0.07-0.68)$ & 66.18 & 183.46 & $0.72(0.49-0.86)$ & 48.21 & 133.62 & $0.53(0.31-0.72)$ & 59.91 & 166.06 \\
\hline & \multicolumn{13}{|c|}{ Symmetry ratio parameters } \\
\hline & speed SR & $0.22(-0.16-0.54)$ & 2.56 & 7.11 & $0.09(-0.24-0.42)$ & 2.06 & 5.72 & $0.56(0.25-0.77)$ & 2.07 & 5.75 & $0.33(0.11-0.57)$ & 2.25 & 6.25 \\
\hline & cadence SR & $0.15(-0.23-0.49)$ & 2.42 & 6.70 & $0.12(-0.22-0.45)$ & 2.53 & 7.02 & $0.73(0.49-0.86)$ & 1.79 & 4.96 & $0.40(0.18-0.63)$ & 2.27 & 6.30 \\
\hline \multirow{6}{*}{$\begin{array}{c}\text { Slight } \\
0.0<\text { ICC }<0.2\end{array}$} & slength SR & $0.13(-0.25-0.47)$ & 1.82 & 5.05 & $0.02(-0.35-0.38)$ & 1.78 & 4.95 & $0.40(0.06-0.66)$ & 1.73 & 4.79 & $0.21(0.00-0.46)$ & 1.78 & 4.93 \\
\hline & swing SR & $0.36(0.00-0.64)$ & 3.00 & 8.32 & $0.54(0.23-0.75)$ & 2.97 & 8.25 & $0.60(0.30-0.79)$ & 2.79 & 7.73 & $0.52(0.30-0.71)$ & 2.93 & 8.11 \\
\hline & stance SR & $0.38(0.02-0.66)$ & 1.74 & 4.83 & $0.56(0.25-0.76)$ & 1.68 & 4.65 & $0.55(0.24-0.76)$ & 1.67 & 4.63 & $0.50(0.29-0.70)$ & 1.70 & 4.71 \\
\hline & LDr SR & $0.62(0.33-0.8)$ & 9.69 & 26.86 & $0.78(0.58-0.89)$ & 7.01 & 19.42 & $0.67(0.41-0.83)$ & 9.04 & 25.06 & $0.69(0.51-0.83)$ & 8.62 & 23.90 \\
\hline & FFr SR & $0.69(0.43-0.84)$ & 3.07 & 8.50 & $0.67(0.40-0.83)$ & 3.03 & 8.41 & $0.83(0.67-0.92)$ & 2.60 & 7.20 & $0.74(0.58-0.86)$ & 2.90 & 8.03 \\
\hline & Pur SR & $0.63(0.34-0.81)$ & 4.92 & 13.63 & $0.59(0.30-0.79)$ & 5.01 & 13.89 & $0.80(0.62-0.90)$ & 3.66 & 10.15 & $0.68(0.50-0.82)$ & 4.55 & 12.62 \\
\hline
\end{tabular}


Table 4. ICC, SEM\% and MDC\% values for spatiotemporal, normalized (N) and symmetry (symmetry index-SI and symmetry ratio-SR) gait parameters obtained in the dual-task condition between the mean of the first and the second trials (T1-T2), the first and the third trials (T1-T3), the second and the third trials (T2-T3) and the means of the three consecutive trials (T1-T2-T3).

\begin{tabular}{|c|c|c|c|c|c|c|c|c|c|c|c|c|c|}
\hline & \multirow[b]{2}{*}{ Parameters } & \multicolumn{3}{|c|}{ T1-T2 } & \multicolumn{3}{|c|}{ T1-T3 } & \multicolumn{3}{|c|}{ T2-T3 } & \multicolumn{3}{|c|}{ T1-2-3 } \\
\hline & & ICC (lb-ub) & SEM(\%) & MDC(\%) & ICC (lb-ub) & SEM(\%) & MDC(\%) & ICC (lb-ub) & SEM(\%) & MDC(\%) & ICC (lb-ub) & SEM(\%) & MDC(\%) \\
\hline \multirow{8}{*}{$\begin{array}{c}\text { Almost } \\
\text { perfect } \\
0.81<\text { ICC } \\
\quad<1\end{array}$} & \multicolumn{13}{|c|}{ Spatiotemporal parameters } \\
\hline & speed & $0.96(0.92-0.98)$ & 3.81 & 10.55 & $0.97(0.95-0.99)$ & 2.93 & 8.13 & $0.98(0.97-0.99)$ & 2.56 & 7.11 & $0.97(0.95-0.99)$ & 3.15 & 7.95 \\
\hline & cadence & $0.96(0.91-0.98)$ & 1.92 & 5.31 & $0.97(0.93-0.98)$ & 1.75 & 4.85 & $0.97(0.94-0.99)$ & 1.71 & 4.75 & $0.97(0.94-0.98)$ & 1.78 & 4.79 \\
\hline & stride length & $0.97(0.94-0.99)$ & 2.44 & 6.76 & $0.98(0.96-0.99)$ & 1.84 & 5.10 & $0.99(0.97-0.99)$ & 1.61 & 4.47 & $0.98(0.96-0.99)$ & 2.03 & 4.94 \\
\hline & DS & $0.84(0.69-0.92)$ & 4.67 & 12.94 & $0.81(0.63-0.91)$ & 4.84 & 13.41 & $0.96(0.91-0.98)$ & 2.72 & 7.53 & $0.87(0.78-0.93)$ & 4.22 & 17.01 \\
\hline & swing & $0.80(0.61-0.9)$ & 1.96 & 5.43 & $0.84(0.69-0.92)$ & 1.47 & 4.07 & $0.91(0.82-0.96)$ & 1.32 & 3.65 & $0.85(0.75-0.92)$ & 1.61 & 5.43 \\
\hline & stance & $0.80(0.61-0.9)$ & 1.17 & 3.25 & $0.84(0.69-0.92)$ & 0.88 & 2.44 & $0.91(0.82-0.96)$ & 0.79 & 2.19 & $0.85(0.75-0.92)$ & 0.96 & 3.33 \\
\hline & LDr & $0.98(0.96-0.99)$ & 3.69 & 10.24 & $0.98(0.95-0.99)$ & 4.21 & 11.68 & $0.98(0.96-0.99)$ & 3.77 & 10.46 & $0.98(0.96-0.99)$ & 3.86 & 17.01 \\
\hline \multirow{6}{*}{$\begin{array}{c}\text { Substantial } \\
0.61<\text { ICC } \\
\quad<0.8\end{array}$} & FFr & $0.97(0.93-0.98)$ & 1.99 & 5.52 & $0.96(0.93-0.98)$ & 1.98 & 5.50 & $0.98(0.96-0.99)$ & 1.50 & 4.17 & $0.97(0.95-0.99)$ & 1.82 & 7.36 \\
\hline & Pur & $0.94(0.87-0.97)$ & 2.66 & 7.36 & $0.94(0.87-0.97)$ & 2.78 & 7.69 & $0.97(0.95-0.99)$ & 1.91 & 5.28 & $0.95(0.91-0.98)$ & 2.46 & 9.08 \\
\hline & \multicolumn{13}{|c|}{ Normalized parameters } \\
\hline & speed $\mathrm{N}$ & $0.96(0.91-0.98)$ & 3.77 & 10.45 & $0.97(0.94-0.99)$ & 2.94 & 8.16 & $0.98(0.96-0.99)$ & 2.58 & 7.16 & $0.97(0.95-0.99)$ & 3.14 & 7.90 \\
\hline & cadence $\mathrm{N}$ & $0.96(0.91-0.98)$ & 1.92 & 5.31 & $0.97(0.93-0.98)$ & 1.75 & 4.85 & $0.97(0.94-0.99)$ & 1.71 & 4.75 & $0.97(0.94-0.98)$ & 1.78 & 4.80 \\
\hline & slength $\mathrm{N}$ & $0.96(0.93-0.98)$ & 2.38 & 6.59 & $0.97(0.95-0.99)$ & 1.86 & 5.15 & $0.98(0.97-0.99)$ & 1.60 & 4.43 & $0.97(0.95-0.99)$ & 2.00 & 4.91 \\
\hline \multirow{6}{*}{$\begin{array}{c}\text { Moderate } \\
0.41<\text { ICC } \\
<0.6\end{array}$} & \multicolumn{13}{|c|}{ Symmetry index parameters } \\
\hline & speed SI & $0.00(-0.37-0.37)$ & 95.83 & 265.62 & $0.05(-0.33-0.41)$ & 76.06 & 210.83 & $0.00(-0.37-0.37)$ & 111.07 & 307.86 & $0.00(-0.18-0.26)$ & 96.94 & 231.93 \\
\hline & cadence SI & $0.08(-0.27-0.42)$ & 129.73 & 359.58 & $0.26(-0.11-0.57)$ & 47.70 & 132.21 & $0.02(-0.30-0.37)$ & 142.81 & 395.84 & $0.06(-0.12-0.30)$ & 127.73 & 434.25 \\
\hline & slength SI & $0.57(0.26-0.78)$ & 44.16 & 122.39 & $0.04(-0.33-0.40)$ & 75.82 & 210.15 & $0.08(-0.29-0.44)$ & 78.02 & 216.26 & $0.19(-0.03-0.45)$ & 67.70 & 230.60 \\
\hline & swing SI & $0.15(-0.23-0.49)$ & 79.54 & 220.48 & $0.48(0.15-0.72)$ & 67.94 & 188.31 & $0.18(-0.16-0.49)$ & 85.30 & 236.44 & $0.30(0.08-0.55)$ & 77.70 & 206.66 \\
\hline & stance SI & $0.10(-0.28-0.45)$ & 81.34 & 225.46 & $0.43(0.10-0.69)$ & 69.38 & 192.30 & $0.20(-0.13-0.51)$ & 82.62 & 229.00 & $0.28(0.06-0.53)$ & 77.61 & 199.54 \\
\hline \multirow{6}{*}{$\begin{array}{c}\quad \text { Fair } \\
0.21<\text { ICC } \\
<0.4\end{array}$} & LDr SI & $0.52(0.19-0.74)$ & 58.55 & 162.30 & $0.34(-0.02-0.62)$ & 65.41 & 181.31 & $0.57(0.25-0.78)$ & 58.65 & 162.57 & $0.48(0.26-0.69)$ & 61.63 & 163.99 \\
\hline & FFr SI & $0.66(0.38-0.82)$ & 47.74 & 132.33 & $0.70(0.45-0.85)$ & 44.11 & 122.25 & $0.79(0.59-0.89)$ & 31.16 & 86.38 & $0.71(0.54-0.84)$ & 41.00 & 144.99 \\
\hline & Pur SI & $0.69(0.44-0.84)$ & 46.02 & 127.55 & $0.76(0.54-0.88)$ & 38.80 & 107.55 & $0.73(0.50-0.87)$ & 37.80 & 104.79 & $0.72(0.55-0.85)$ & 41.30 & 166.06 \\
\hline & \multicolumn{13}{|c|}{ Symmetry ratio parameters } \\
\hline & speed SR & $0.23(-0.15-0.55)$ & 1.89 & 5.25 & $0.10(-0.27-0.45)$ & 1.46 & 4.04 & $0.26(-0.12-0.57)$ & 1.92 & 5.34 & $0.21(-0.01-0.47)$ & 1.77 & 6.25 \\
\hline & cadence SR & $0.37(0.01-0.65)$ & 1.50 & 4.15 & $0.51(0.18-0.74)$ & 0.58 & 1.60 & $0.00(-0.37-0.37)$ & 1.87 & 5.18 & $0.20(-0.02-0.46)$ & 1.43 & 6.30 \\
\hline \multirow{6}{*}{$\begin{array}{c}\text { Slight } \\
0.0<\text { ICC } \\
<0.2\end{array}$} & slength SR & $0.20(-0.18-0.52)$ & 1.13 & 3.13 & $0.00(-0.37-0.37)$ & 1.45 & 4.03 & $0.31(-0.06-0.61)$ & 1.26 & 3.50 & $0.16(-0.05-0.42)$ & 1.29 & 4.93 \\
\hline & swing SR & $0.13(-0.25-0.47)$ & 3.05 & 8.46 & $0.50(0.17-0.73)$ & 3.39 & 9.39 & $0.40(0.05-0.67)$ & 3.45 & 9.57 & $0.40(0.18-0.63)$ & 3.25 & 8.11 \\
\hline & stance SR & $0.11(-0.26-0.46)$ & 1.85 & 5.12 & $0.43(0.09-0.69)$ & 2.06 & 5.70 & $0.48(0.15-0.72)$ & 1.84 & 5.11 & $0.40(0.17-0.63)$ & 1.89 & 4.71 \\
\hline & LDr SR & $0.78(0.57-0.89)$ & 6.18 & 17.12 & $0.68(0.41-0.84)$ & 9.00 & 24.95 & $0.77(0.56-0.88)$ & 7.28 & 20.17 & $0.74(0.58-0.86)$ & 7.52 & 23.90 \\
\hline & FFr SR & $0.78(0.58-0.89)$ & 2.85 & 7.89 & $0.83(0.67-0.92)$ & 2.55 & 7.08 & $0.87(0.74-0.94)$ & 2.16 & 5.98 & $0.83(0.71-0.91)$ & 2.52 & 8.03 \\
\hline & Pur SR & $0.67(0.41-0.83)$ & 4.17 & 11.57 & $0.77(0.56-0.89)$ & 3.30 & 9.14 & $0.88(0.76-0.94)$ & 2.47 & 6.83 & $0.77(0.63-0.88)$ & 3.38 & 12.62 \\
\hline
\end{tabular}


All ICC values for spatiotemporal and normalized gait parameters were above 0.81 in single- and dual-task conditions, except for swing, stance, and double support percentage in single-task condition where ICC values were above 0.61 (Tables 3 and 4). MDC \% values were comprised between $2.19 \%$ and $22.95 \%$ for these parameters in single- and dual-task conditions.

Symmetry ratio parameters reliability was low but was acceptable when using the 2nd and 3rd trials $(0.40<\mathrm{ICC}<0.83,4.63<\mathrm{MDC} \%<25.06$ in single-task; $0.00<\mathrm{ICC}<0.79,3.50<\mathrm{MDC} \%<20.17$ in dual-task), while symmetry index parameters reliability was weaker $(0.25<$ ICC $<0.75,121.08<$ MDC $\%<298.61$ in single-task; $0.00<$ ICC $<0.79,86.38<$ MDC $\%<395.84$ in dual-task).

Supplementary Tables S1 and S2 present limits of agreement, SEM and MDC values for the spatiotemporal and symmetry parameters in single- and dual-task conditions.

Supplementary Figure S1 presents Bland and Altman plots for speed, cadence, and stride length parameters in single- and dual-task conditions.

\subsection{Intra-Session Relative and Absolute Reliability of Dual Task Effects Parameters}

Tables 5 and 6 show respectively means and standard deviations of dual task effects and dual task effects percentages of spatiotemporal gait parameters for each trial, the mean of the 3 trials, and $p$-values obtained from RM-ANOVA with differences between trials.

Table 5. Mean and standard deviation (SD) of the dual task effects (DTE) for each trial and for the three trials.

\begin{tabular}{|c|c|c|c|c|c|c|c|c|c|c|c|}
\hline & \multicolumn{2}{|c|}{ Trial 1} & \multicolumn{2}{|c|}{ Trial 2} & \multicolumn{2}{|c|}{ Trial 3} & \multicolumn{2}{|c|}{ Trial 1-2-3 } & \multirow{2}{*}{$\begin{array}{l}\text { T1-T2 } \\
p \text {-Value }\end{array}$} & \multirow{2}{*}{$\begin{array}{l}\text { T1-T3 } \\
p \text {-Value }\end{array}$} & \multirow{2}{*}{$\begin{array}{c}\text { T2-T3 } \\
p \text {-Value }\end{array}$} \\
\hline & Mean & SD & Mean & SD & Mean & SD & Mean & SD & & & \\
\hline Speed DTE & 0.06 & 0.10 & 0.08 & 0.12 & 0.09 & 0.11 & 0.08 & 0.11 & 0.94 & 0.71 & 0.89 \\
\hline Cadence DTE & 0.34 & 4.17 & 1.62 & 4.04 & 2.15 & 5.17 & 1.35 & 4.47 & 0.65 & 0.42 & 0.93 \\
\hline Stride length DTE & 0.07 & 0.08 & 0.07 & 0.09 & 0.07 & 0.08 & 0.07 & 0.08 & 0.99 & 0.96 & 0.99 \\
\hline Double support ${ }^{\mathrm{a}} \mathrm{DTE}$ & -1.32 & 2.61 & 0.72 & 7.05 & -1.51 & 2.43 & -0.71 & 4.57 & 0.35 & 0.99 & 0.29 \\
\hline Swing DTE & 0.56 & 1.03 & 0.48 & 1.32 & 0.57 & 1.25 & 0.54 & 1.18 & 0.97 & 1.00 & 0.97 \\
\hline Stance ${ }^{a}$ DTE & -0.56 & 1.03 & -0.48 & 1.32 & -0.57 & 1.25 & -0.54 & 1.18 & 0.97 & 1.00 & 0.97 \\
\hline Load $^{\mathrm{a}}$ DTE & -0.09 & 1.64 & -0.10 & 1.61 & 0.20 & 1.07 & 0.00 & 1.44 & 1.00 & 0.82 & 0.81 \\
\hline Foot flat DTE & 0.23 & 2.54 & -0.28 & 2.96 & -0.41 & 2.94 & -0.15 & 2.78 & 0.84 & 0.76 & 0.99 \\
\hline Push ${ }^{a}$ DTE & -0.14 & 2.15 & 0.38 & 2.21 & 0.21 & 2.23 & 0.14 & 2.17 & 0.74 & 0.87 & 0.97 \\
\hline
\end{tabular}

a a negative value is associated to worse performance in the dual-task condition.

Table 6. Mean and standard deviation (SD) of the dual task effects percentage (DTE\%) for each trial and for the three trials.

\begin{tabular}{|c|c|c|c|c|c|c|c|c|c|c|c|}
\hline & \multicolumn{2}{|c|}{ Trial 1} & \multicolumn{2}{|c|}{ Trial 2} & \multicolumn{2}{|c|}{ Trial 3} & \multicolumn{2}{|c|}{ Trial 1-2-3 } & \multirow{2}{*}{$\begin{array}{l}\text { T1-T2 } \\
p \text {-Value }\end{array}$} & \multirow{2}{*}{$\begin{array}{l}\text { T1-T3 } \\
p \text {-Value }\end{array}$} & \multirow{2}{*}{$\begin{array}{c}\text { T2-T3 } \\
p \text {-Value }\end{array}$} \\
\hline & Mean & SD & Mean & SD & Mean & SD & Mean & SD & & & \\
\hline Speed DTE\% & 5.03 & 8.43 & 6.51 & 9.64 & 7.69 & 9.57 & 6.38 & 9.12 & 0.87 & 0.64 & 0.92 \\
\hline Cadence DTE\% & 0.23 & 3.89 & 1.60 & 3.75 & 2.17 & 5.13 & 1.31 & 4.30 & 0.59 & 0.35 & 0.91 \\
\hline Stride length DTE\% & 4.96 & 5.66 & 5.43 & 6.64 & 5.52 & 5.76 & 5.30 & 5.93 & 0.97 & 0.95 & 1.00 \\
\hline $\begin{array}{l}\text { Double support }{ }^{\text {a }} \\
\text { DTE\% }\end{array}$ & -7.04 & 13.86 & -3.45 & 10.11 & -6.77 & 10.62 & -5.82 & 11.62 & 0.62 & 1.00 & 0.67 \\
\hline Swing DTE\% & 1.42 & 2.69 & 1.27 & 3.49 & 1.45 & 3.32 & 1.38 & 3.12 & 0.99 & 1.00 & 0.98 \\
\hline Stance ${ }^{a}$ DTE\% & -0.94 & 1.68 & -0.77 & 2.12 & -0.93 & 2.02 & -0.88 & 1.91 & 0.96 & 1.00 & 0.96 \\
\hline Load ${ }^{a}$ DTE $\%$ & -0.64 & 11.30 & -0.46 & 11.80 & 1.45 & 8.39 & 0.11 & 10.47 & 1.00 & 0.81 & 0.85 \\
\hline Foot flat DTE\% & 0.22 & 4.75 & -0.48 & 5.70 & -0.78 & 5.61 & -0.34 & 5.28 & 0.91 & 0.83 & 0.98 \\
\hline Push ${ }^{a}$ DTE\% & -0.79 & 6.91 & 1.23 & 6.45 & 0.61 & 6.56 & 0.33 & 6.59 & 0.61 & 0.79 & 0.96 \\
\hline
\end{tabular}

a a negative value is associated to worse performance in the dual-task condition.

Tables 7 and 8 show reliability of DTE and DTE\% for each spatiotemporal gait parameters using ICC, SEM, and MDC. 
Table 7. ICC. SEM and MDC for dual task effects parameters (DTE) between the mean of the first and the second trials (T1-T2), the first and the third trials (T1-T3), the second and the third trials (T2-T3) and the means of the three consecutive trials (T1-T2-T3).

\begin{tabular}{|c|c|c|c|c|c|c|c|c|c|c|c|c|c|}
\hline & \multirow{2}{*}{$\begin{array}{c}\text { Trials } \\
\text { Parameters }\end{array}$} & \multicolumn{3}{|c|}{ T1-T2 } & \multicolumn{3}{|c|}{ T1-T3 } & \multicolumn{3}{|c|}{ T2-T3 } & \multicolumn{3}{|c|}{ T1-2-3 } \\
\hline & & ICC $(95 \%$ CI) & SEM $\%$ & MDC\% & ICC $(95 \%$ CI) & SEM $\%$ & MDC\% & ICC (95\% CI) & SEM $\%$ & MDC\% & ICC (95\% CI) & SEM $\%$ & MDC\% \\
\hline $\begin{array}{l}\text { Almost perfect } \\
0.81<\text { ICC }<1\end{array}$ & $\begin{array}{l}\text { Speed DTE } \\
\text { Cadence DTE }\end{array}$ & $\begin{array}{l}0.79(0.55-0.91) \\
0.53(0.22-0.75)\end{array}$ & $\begin{array}{l}71.78 \\
290.97\end{array}$ & $\begin{array}{l}198.96 \\
806.53\end{array}$ & $\begin{array}{l}0.79(0.55-0.91) \\
0.61(0.32-0.80)\end{array}$ & $\begin{array}{r}62.35 \\
240.02\end{array}$ & $\begin{array}{l}172.82 \\
665.31\end{array}$ & $\begin{array}{l}0.82(0.60-0.92) \\
0.63(0.35-0.81)\end{array}$ & $\begin{array}{l}57.47 \\
147.37\end{array}$ & $\begin{array}{l}159.31 \\
408.48\end{array}$ & $\begin{array}{l}0.80(0.64-0.91) \\
0.60(0.40-0.77)\end{array}$ & $\begin{array}{r}62.94 \\
209.01\end{array}$ & $\begin{array}{l}174.45 \\
579.35\end{array}$ \\
\hline $\begin{array}{c}\text { Substantial } \\
0.61<\text { ICC }<0.8\end{array}$ & $\begin{array}{l}\text { Stride length DTE } \\
\text { Double support DTE }\end{array}$ & $\begin{array}{c}0.78(0.53-0.91) \\
0.18(-0.25-0.56)\end{array}$ & $\begin{array}{l}397.53 \\
200.94\end{array}$ & $\begin{array}{c}150.60 \\
4042.96\end{array}$ & $\begin{array}{c}0.80(0.57-0.92) \\
0.01(-0.42-0.44)\end{array}$ & $\begin{array}{l}351.19 \\
185.28\end{array}$ & $\begin{array}{l}130.35 \\
485.97\end{array}$ & $\begin{array}{c}0.81(0.59-0.92) \\
0.01(-0.42-0.44)\end{array}$ & $\begin{array}{l}202.95 \\
169.57\end{array}$ & $\begin{array}{l}132.60 \\
485.97\end{array}$ & $\begin{array}{l}0.80(0.63-0.91) \\
0.19(-0.06-0.49)\end{array}$ & $\begin{array}{l}310.60 \\
186.45\end{array}$ & $\begin{array}{c}137.53 \\
1597.28\end{array}$ \\
\hline $\begin{array}{c}\text { Moderate } \\
0.41<\text { ICC }<0.6\end{array}$ & $\begin{array}{l}\text { Swing DTE } \\
\text { Stance DTE }\end{array}$ & $\begin{array}{l}0.18(-0.27-0.57) \\
0.18(-0.27-0.57)\end{array}$ & $\begin{array}{c}54.33 \\
200.94\end{array}$ & $\begin{array}{l}556.97 \\
556.97\end{array}$ & $\begin{array}{l}0.14(-0.31-0.54) \\
0.14(-0.31-0.54)\end{array}$ & $\begin{array}{l}47.03 \\
185.28\end{array}$ & $\begin{array}{l}513.57 \\
513.57\end{array}$ & $\begin{array}{l}0.51(0.11-0.77) \\
0.51(0.11-0.77)\end{array}$ & $\begin{array}{l}47.84 \\
169.57\end{array}$ & $\begin{array}{l}470.02 \\
470.02\end{array}$ & $\begin{array}{l}0.28(0.01-0.58) \\
0.28(0.01-0.58)\end{array}$ & $\begin{array}{c}49.62 \\
186.45\end{array}$ & $\begin{array}{l}516.80 \\
516.80\end{array}$ \\
\hline $\begin{array}{c}\text { Fair } \\
0.21<\text { ICC }<0.4\end{array}$ & Load DTE & $0.65(0.31-0.85)$ & 1458.57 & 2869.52 & $0.42(-0.01-0.72)$ & 175.32 & 5486.33 & $0.44(0.02-0.73)$ & 175.32 & 5446.06 & $0.52(0.26-0.75)$ & 576.25 & $69,420.96$ \\
\hline $\begin{array}{c}\text { Slight } \\
0.0<\text { ICC }<0.2\end{array}$ & $\begin{array}{l}\text { Foot flat DTE } \\
\text { Push DTE }\end{array}$ & $\begin{array}{l}0.35(-0.09-0.68) \\
0.31(-0.13-0.66)\end{array}$ & $\begin{array}{c}1035.23 \\
10,333.41\end{array}$ & $\begin{array}{c}28,642.76 \\
4432.51\end{array}$ & $\begin{array}{c}0.43(0.00-0.73) \\
0.28(-0.17-0.63)\end{array}$ & $\begin{array}{l}1979.30 \\
2534.27\end{array}$ & $\begin{array}{c}7024.64 \\
18,170.05\end{array}$ & $\begin{array}{l}0.78(0.54-0.91) \\
0.72(0.42-0.88)\end{array}$ & $\begin{array}{c}1964.77 \\
391.96\end{array}$ & $\begin{array}{l}1086.47 \\
1100.67\end{array}$ & $\begin{array}{l}0.53(0.27-0.76) \\
0.44(0.16-0.69)\end{array}$ & $\begin{array}{c}25,044.91 \\
1286.43\end{array}$ & $\begin{array}{l}3565.81 \\
3145.73\end{array}$ \\
\hline
\end{tabular}

Table 8. ICC. SEM and MDC for dual task effects percent parameters (DTE\%) between the mean of the first and second trials (T1-T2). the first and the third trials (T1-T3). the second and the third trials (T2-T3) and the means of the three consecutive trials (T1-T2-T3).

\begin{tabular}{|c|c|c|c|c|c|c|c|c|c|c|c|c|c|}
\hline & \multirow{2}{*}{$\begin{array}{c}\text { Trials } \\
\text { Parameters }\end{array}$} & \multicolumn{3}{|c|}{ T1-T2 } & \multicolumn{3}{|c|}{ T1-T3 } & \multicolumn{3}{|c|}{ T2-T3 } & \multicolumn{3}{|c|}{ T1-2-3 } \\
\hline & & ICC $(95 \% \mathrm{CI})$ & SEM $\%$ & MDC\% & ICC $(95 \% \mathrm{CI})$ & SEM $\%$ & MDC\% & ICC (95\% CI) & SEM $\%$ & MDC $\%$ & ICC $(95 \% \mathrm{CI})$ & SEM $\%$ & MDC\% \\
\hline $\begin{array}{l}\text { Almost perfect } \\
0.81<\text { ICC }<1\end{array}$ & $\begin{array}{l}\text { Speed DTE\% } \\
\text { Cadence DTE\% }\end{array}$ & $\begin{array}{l}0.75(0.47-0.89) \\
0.48(0.09-0.75)\end{array}$ & $\begin{array}{c}78.32 \\
307.94\end{array}$ & $\begin{array}{l}217.09 \\
853.56\end{array}$ & $\begin{array}{l}0.75(0.48-0.89) \\
0.50(0.11-0.77)\end{array}$ & $\begin{array}{r}70.58 \\
274.85\end{array}$ & $\begin{array}{l}195.64 \\
761.86\end{array}$ & $\begin{array}{l}0.84(0.64-0.93) \\
0.63(0.28-0.84)\end{array}$ & $\begin{array}{c}54.24 \\
143.21\end{array}$ & $\begin{array}{l}150.35 \\
396.96\end{array}$ & $\begin{array}{l}0.78(0.61-0.90) \\
0.55(0.29-0.76)\end{array}$ & $\begin{array}{c}66.64 \\
220.50\end{array}$ & $\begin{array}{l}184.71 \\
611.20\end{array}$ \\
\hline $\begin{array}{c}\text { Substantial } \\
0.61<\text { ICC }<0.8\end{array}$ & $\begin{array}{l}\text { Stride length DTE\% } \\
\text { Double support DTE\% }\end{array}$ & $\begin{array}{c}0.79(0.55-0.91) \\
0.35(-0.08-0.68)\end{array}$ & $\begin{array}{l}358.55 \\
195.41\end{array}$ & $\begin{array}{l}148.72 \\
510.94\end{array}$ & $\begin{array}{c}0.82(0.6-0.93) \\
0.00(-0.43-0.43)\end{array}$ & $\begin{array}{l}286.69 \\
180.82\end{array}$ & $\begin{array}{l}125.05 \\
490.73\end{array}$ & $\begin{array}{l}0.85(0.66-0.94) \\
0.59(0.22-0.81)\end{array}$ & $\begin{array}{l}201.32 \\
166.43\end{array}$ & $\begin{array}{l}121.19 \\
358.26\end{array}$ & $\begin{array}{c}0.82(0.67-0.92) \\
0.25(-0.01-0.55)\end{array}$ & $\begin{array}{l}279.50 \\
181.99\end{array}$ & $\begin{array}{l}131.29 \\
479.41\end{array}$ \\
\hline $\begin{array}{c}\text { Moderate } \\
0.41<\text { ICC }<0.6\end{array}$ & $\begin{array}{l}\text { Swing DTE\% } \\
\text { Stance DTE\% }\end{array}$ & $\begin{array}{l}0.14(-0.31-0.54) \\
0.21(-0.24-0.59)\end{array}$ & $\begin{array}{r}53.65 \\
210.99\end{array}$ & $\begin{array}{l}584.83 \\
541.65\end{array}$ & $\begin{array}{l}0.13(-0.32-0.53) \\
0.14(-0.31-0.54)\end{array}$ & $\begin{array}{l}45.11 \\
193.69\end{array}$ & $\begin{array}{l}536.88 \\
501.20\end{array}$ & $\begin{array}{l}0.49(0.09-0.76) \\
0.52(0.12-0.78)\end{array}$ & $\begin{array}{c}43.72 \\
176.00\end{array}$ & $\begin{array}{l}487.85 \\
461.31\end{array}$ & $\begin{array}{c}0.26(-0.01-0.56) \\
0.30(0.03-0.59)\end{array}$ & $\begin{array}{c}47.36 \\
194.73\end{array}$ & $\begin{array}{l}539.75 \\
504.46\end{array}$ \\
\hline $\begin{array}{c}\text { Fair } \\
0.21<\text { ICC }<0.4\end{array}$ & Load DTE $\%$ & $0.63(0.29-0.84)$ & 184.33 & 3484.78 & 0. & 177.04 & 5058.62 & $-0.74)$ & 129.25 & 4177.14 & 76) & 172.96 & 28 \\
\hline Sligh & Foot flat DTE\% & $0.35(-$ & 1257.20 & 9639.47 & 0.42 & 10.629 & 405 & s & 15 & 55 & & 73 & 2982.74 \\
\hline $0.0<$ ICC $<0.2$ & Push DTE\% & $0.75(0.47-0.89)$ & 3477.62 & 217.09 & $0.75(0.48-0.89)$ & 1462.86 & 195.64 & $0.84(0.64-0.93)$ & 411.51 & 150.35 & $0.78(0.61-0.90)$ & 1076.08 & 184.71 \\
\hline
\end{tabular}


ICC values for the speed, stride length DTE and DTE\% were above 0.61 .

ICC values for the cadence, load ratio DTE and DTE\% were comprised between 0.41 and 0.8 .

ICC values for the foot flat and push ratios, and swing, stance, and double support percentage DTE and DTE\% were under 0.6.

Swing, stance, and double support percentages DTE\% showed better reliability for trials 2-3 with ICC values comprised between 0.49 and 0.59 . DTE\% parameters showed better relative reliability (i.e., higher ICC) than DTE parameters.

Supplementary Tables S3 and S4present limits of agreement, SEM, and MDC values for DTE and DTE $\%$ of spatiotemporal gait parameters.

Supplementary Figure S1 presents Bland and Altman plots for speed, cadence, and stride length DTE parameters.

\subsection{Number of Trials to Ensure Reliable Measurements}

The mean values of gait parameters obtained in the single- and dual-task conditions were not significantly different between trials whatever the conducted comparisons with $p$-values ranging from 0.73 to 1.0 (Tables 1 and 2). Same results are observed with DTE and DTE\% where no statistically differences were found.

The comparisons of means of ICC between trials showed that slightly higher ICC and lower SEM and MDC were obtained when pooling gait parameters from the second and the third trials, compared to the first and second trials, to the first and third trials, or to the three trials in the single- and the dual-task conditions.

\section{Discussion}

The study demonstrated excellent intra-session reliability of spatiotemporal and normalized gait parameters assessed by foot-worn inertial sensors under single and dual-task walking in patients with axSpA. This study also gives SEM and MDC of basic spatiotemporal gait parameters, in single- and dual-task conditions, for this population. The reliability was better for the second and the third trials, suggesting that the first trial could be considered as a warming before real assessments. The present study demonstrated low reliability of symmetry gait parameters and dual task effects obtained from spatiotemporal gait parameters.

\subsection{Reliability of Gait Parameters in Patients with axSpA}

Gait parameters measured in patients with axSpA were reliable in both single- and dual-task conditions. Although the population is different, this result is in line with previous reviews reporting good to excellent reliability of single and dual-task assessments in older adults [67] or patients with neurological conditions [68]. We showed that relative reliability of spatiotemporal and gait parameters obtained in single- and dual-task conditions, as assessed by ICC, was considered as excellent for most of the parameters $(0.81<$ ICC $<0.98)$. The swing $(\%)$, stance $(\%)$ and double support $(\%)$ times were slightly less reliable than the other spatiotemporal gait parameters, but showed still moderate to perfect reliability $(0.59<$ ICC $<0.90)$. Symmetry gait parameters showed low relative reliability (Tables 5 and 6) in line with results reported from previous studies with healthy participants [3,69-71]. Symmetry ratio presented better reliability than symmetry index, notably for the second and third trials with ICC comprised between 0.40 and 0.83 considered as moderate to substantial reliability.

SEM and MDC were acceptable in single- and dual-task conditions. For spatiotemporal and symmetry ratio parameters, a change of $2-25 \%$ of performance $(2.19<\mathrm{MDC} \%<25.06)$ could be identified but lower absolute reliability was found for symmetry index parameters $(86.38<\mathrm{MDC} \%<395.84)$. These results are comparable to those reported in recent studies in which Physilog ${ }^{\circledR}$ inertial sensors were also to measured spatiotemporal gait parameters [52,72]. Indeed, Lefeber et al. in 2019 reported a test retest reliability of gait parameters at self-selected comfortable speed on a 12-m walkway, with 20-min break between sessions in patients after a stroke using foot-worn sensors. Comparable 
results were reported with ICC values ranging from 0.546 to 0.985 , with lower reliability for stance and swing phase percentage (0.547) [52]. Lower reliability of these parameters was explained by less apparent negative peaks in foot pitch angular velocity related to weakened foot lifters, leading to lower detection of heel strike and/or toe off. Standard error of measurement (SEM) observed in the present study (SEM values comprised from 0.01 to 1.93 in single task, Supplementary Table S1) were lower than those reported by Lefeber et al. in patients post stroke [52] with SEM values comprised between 0.038 and 4.344. Most of the higher values of SEM, obtained in Lefeber et al.study [52], were those of paretic leg of patients with stroke and was partly explained by poor heel strike or toe-off detection of this leg by inertial sensors leading to higher error of measurement. Another study used Physilog ${ }^{\circledR}$ inertial sensors attached to each shank in patients with diabetes walking on a 50-m tarred pathway and calculated the inter- and intra-session reliability at preferred walking speed [72]. Intra-session relative reliability was comprised between 0.938 and 0.976 for cadence, speed, gait cycle time, stride length, stance phase and double support. However, the intra-session absolute reliability was not reported [72].

\subsection{Reliability of Dual Task Effects of Gait Parameters in Patients with axSpA}

Dual task effects are used to assess the cost of performing another task while walking [51,61]. In the present study, we tested the intra-session reliability of DTE and DTE\% in patients with axSpA. Although DTE parameter is frequently used as an outcome in gait studies $[13,17,51,73]$, it is interesting to note that, to the best of our knowledge, its reliability has never been studied in patients with axSpA. Our results showed that the reliability of DTE and DTE\% was variable and dependent of the parameter used. The relative reliability was considered as poor to substantial $(0.00<$ ICC $<0.82)$ for DTE, and was better for DTE\% $(0.00<$ ICC > 0.85). The absolute reliability of DTE ranged respectively from 0.03 to 4.79 for SEM, and from 0.09 to 13.29 for MDC, and of DTE\% from 1.41 to 12.23 for SEM and 3.92 to 33.89 for MDC. If the inter-session reliability of DTE parameters has already been partly assessed in healthy individuals $[66,67,74]$ and pathological populations $[13,62,66,68,75-77]$, only few studies have assessed the intra-session reliability of DTE parameters, only in healthy populations [78]. In this study [78], two trials were performed when walking on a 30-m walkway in single- and dual-task (texting) conditions using body worn sensor in 31 healthy young adults (age: 22.5 $\pm 2.1,19$ females). Gait speed and texting DTE reliability were calculated and relative reliability (ICC) was comprised between 0.76 and 0.91 for gait speed DTE, and 0.03 and 0.22 for texting DTE. Absolute reliability (SEM) was comprised between 3.15 and 4.29 for gait speed DTE and 26.02 and 31.06 for texting DTE. The low relative reliability of DTE could be explained by the addition of measurement errors of single- and dual-task that tend to inflation when combined to obtain DTE [66]. Patients with axSpA we have tested in the present study were asked not to prioritize one task (gait or carry the full cup of water) and may have presented several strategies managing the dual task [67]. Indeed, better inter-session reliability was found when healthy subjects were given instructions about how to prioritize their attention [78]. Taken together, our results suggest that researchers and clinicians should be aware of the poor reliability of DTE among axSpA, particularly for some spatiotemporal gait parameters (e.g., swing, stance, and double support percentages in the present study) and should use speed or stride length which had better DTE reliability.

\subsection{Number of Trials to Ensure Reliable Measurements}

The reliability of gait parameters was substantial to perfect in the single- and the dual-task conditions. Interestingly, we further observed better ICC (i.e., higher), SEM and MDC (i.e., lower) when using gait parameters calculated during the second and the third trials. The reliability was lower when using the first and second trials, the first and third or the three trials. Most of studies on gait used the average of three trials in various populations (e.g., healthy $[50,79]$ or pathological [80]), but the number of trials necessary to obtain reliable measurements has been poorly investigated $[28,81]$.

Our results showed that the first trial appears less reliable than the mean of the second and third trials. This is in line with results reported in previous studies $[28,81]$. For instance, in the study 
of Brach et al. conducted with 558 healthy older adults, the reliability of gait variability was better when calculated from two 4-m walks than from a single 4-m walk using a Gait Mat II system [81]. These authors explained that by increasing the number of steps in the analysis, it increases the sample size, producing more accurate measure of the real width of the distribution [81]. As well, in the study of Fransen et al. performed on 41 patients with knee osteoarthritis, authors reported greater reliability when using trials 2 and 3 compared to trials 1 and 2 [28]. They also found that the reliability was not increased by increasing the number of trials from two to three, or from two to five. Following these results, these authors advised to allow a familiarization trial at each self-selected walking speed [28].

Taken together, these results and ours suggest that the mean of two trials should be used for better reliability of spatiotemporal gait parameters and the first trial should be used, like other studies, as a warming trial (e.g., "The first trial was an exercise to get accustomed to walking protocol and that was not included in further signal analysis" [3]).

\subsection{Limitations and Perspectives}

This study presents several limitations. First, axSpA being a heterogeneous disease [82], the included population ( $n=20$; disease duration: 11 years) could hence not represent the entire population of axSpA. Moreover, patients presented pain at time at measurements (pain intensity: 3.3) that has been previously reported to influence spatiotemporal gait parameters [83]. Furthermore, only one concurrent and non-verbal task (i.e., a manual task) has been used to assess the reliability of gait performance in dual-task conditions, while gait alterations are dependent of the complexity of the concurrent task [84]. Further, the reliability in the present study was assessed during one session (intra- or within-session reliability), which does not allow the calculation of inter-session reliability.

Further studies should focus on the assessment of the reliability of gait parameters obtained from foot-worn inertial sensors in other clinical tests that are commonly used in patients with axSpA, like the timed up and go or the 6-min walk test [41,85], and for the assessment of inter-session reliability.

\section{Conclusions}

This study showed that spatiotemporal and symmetry ratio gait parameters measured with foot-worn inertial sensors in patients with axSpA had good reliability in both single- and dual-task walking conditions. This study also provides SEM and MDC values for this population that could be used for future interventions on gait performance. Our findings further suggest that the first trial of each condition could be considered as a warming trial, and the second and the third trials should be averaged to ensure the best reliability of gait measurements in patients with axSpA. Symmetry index and dual task effects parameters reliability was considered as poor, suggesting that clinicians and researchers should be careful when using these outcomes.

Supplementary Materials: The following are available online at http://www.mdpi.com/1424-8220/20/22/6453/s1, Table S1. LOA, SEM and MDC values for trial 1 and 2, 1 and 3, and 2 and 3 for spatiotemporal, normalized and symmetry gait parameters in single-task condition; Table S2. LOA, SEM and MDC values for trial 1 and 2, 1 and 3, and 2 and 3 for spatiotemporal, normalized and symmetry gait parameters in dual-task condition; Table S3. LOA, SEM and MDC values for trial 1 and 2, 1 and 3, and 2 and 3 for DTE parameters; Table S4. LOA, SEM and MDC values for trial 1 and 2, 1 and 3, and 2 and 3 for DTE\% parameters; Figure S1. Bland and Altman plots for speed, cadence and stride length parameters in single task, dual task, and for DTE parameters.

Author Contributions: Conceptualization, J.V., A.B., P.G., and N.V.; Data curation, J.S.; Formal analysis, J.S., R.B., and N.V.; Funding acquisition, J.V., P.G., and N.V.; Investigation, J.S., J.V., A.B., and P.G.; Methodology, J.S. and N.V.; Supervision, N.V.; Writing—original draft, J.S.; Writing—review \& editing, J.V., R.B., A.B., P.G., and N.V. All authors have read and agreed to the published version of the manuscript. 
Funding: This study is part of the Ph.D. thesis of the first author Julie Soulard (University Grenoble Alpes, AGEIS, France \& Grenoble Alps University Hospital, Grenoble, France). Part of the results of this study was presented at the World congress for neurorehabilitation jointly with the congress of the French society of physical and rehabilitation medicine (7-11 October 2020). This work is supported by the French Ministry of Health and Solidarity with the "Nursing and Paramedical Hospital Research Program" year 2016 (PHRIP-16-0528) and by the French National Research Agency in the framework of the "Investissements d'avenir" program (ANR-10-AIRT-05 and ANR-15-IDEX-02). The sponsors had no involvement in the design of the study, the collection, analysis, and interpretation of data, and in writing the manuscript. This work further forms part of a broader translational and interdisciplinary research program, GaitAlps (N.V.).

Acknowledgments: The authors would like to thank the participants of the study and the FOLOMI team (J. Soulard, N. Vuillerme, P. Gaudin, L. Grange, A. Baillet, J-L Cracowski, R. Juvin, and J. Vaillant), for conceiving and designing the FOLOMI study.

Conflicts of Interest: The authors declare no conflict of interest. No benefits in any form have been received or will be received from a commercial party related directly or indirectly to the subject of this work. None of the authors have any links to companies or manufacturers who would benefit from this work. The funders had no role in the design of the study; in the collection, analyses, or interpretation of data; in the writing of the manuscript, or in the decision to publish the results.

Ethics Approval and Consent to Participate: The study was approved by local ethic committee (CPP Ile De France 1, RCB: 2017-A03468-45, date of agreement: 17 July 2018, Last version: V6.0, 2020, 17 June). The study is registered on ClinicalTrials.gov, with the following ID: NCT03761212 and follow the SPIRIT checklist. Written informed consent were obtained from all participants by the physiotherapist or a doctor. Any modification to the initial protocol will be presented to the local ethic committee and has to be accepted before application and registered ClinicalTrials.gov.

Data Availability: The data of the present manuscript can be available on demand to the corresponding author.

\section{Abbreviations}

$\begin{array}{ll}\text { axSpA } & \text { Axial Spondyloarthritis } \\ \text { DS } & \text { double support } \\ \text { DTE } & \text { Dual task effects } \\ \text { FFr } & \text { Foot flat ratio } \\ \text { FOLOMI } & \text { Function Locomotion Measurement Inflammation } \\ \text { ICC } & \text { Intraclass Correlation Coefficient } \\ \text { LDr } & \text { Load Ratio } \\ \text { MDC } & \text { Minimum Detectable Change } \\ \text { N } & \text { normalized } \\ \text { PUr } & \text { Push ratio } \\ \text { SEM } & \text { Standard Error of Measurement } \\ \text { SI } & \text { symmetry index } \\ \text { SR } & \text { symmetry ratio }\end{array}$

\section{References}

1. Bouisset, S.; Maton, B. Muscles, Posture et Mouvement: Bases et Applications de la Méthode Electromyographique; Hermann: Paris, France, 1996; ISBN 978-2-7056-6191-5.

2. Warren, M.; Ganley, K.J.; Pohl, P.S. The association between social participation and lower extremity muscle strength, balance, and gait speed in US adults. Prev. Med. Rep. 2016, 4, 142-147. [CrossRef]

3. Byun, S.; Han, J.W.; Kim, T.H.; Kim, K.W. Test-Retest Reliability and Concurrent Validity of a Single Tri-Axial Accelerometer-Based Gait Analysis in Older Adults with Normal Cognition. PLoS ONE 2016, 11, e0158956. [CrossRef] [PubMed]

4. Marques, N.R.; Spinoso, D.H.; Cardoso, B.C.; Moreno, V.C.; Kuroda, M.H.; Navega, M.T. Is it possible to predict falls in older adults using gait kinematics? Clin. Biomech. 2018, 59, 15-18. [CrossRef] [PubMed]

5. Beauchet, O.; Launay, C.P.; Sekhon, H.; Barthelemy, J.-C.; Roche, F.; Chabot, J.; Levinoff, E.J.; Allali, G. Association of increased gait variability while dual tasking and cognitive decline: Results from a prospective longitudinal cohort pilot study. GeroScience 2017, 39, 439-445. [CrossRef]

6. Veronese, N.; Stubbs, B.; Volpato, S.; Zuliani, G.; Maggi, S.; Cesari, M.; Lipnicki, D.M.; Smith, L.; Schofield, P.; Firth, J.; et al. Association between gait speed with mortality, cardiovascular disease and cancer: 
A systematic review and meta-analysis of prospective cohort studies. J. Am. Med. Dir. Assoc. 2018, 19, 981-988.e7. [CrossRef] [PubMed]

7. Iosa, M.; Picerno, P.; Paolucci, S.; Morone, G. Wearable inertial sensors for human movement analysis. Expert Rev. Med. Devices 2016, 13, 641-659. [CrossRef]

8. Mirelman, A.; Shema, S.; Maidan, I.; Hausdorff, J.M. Gait. Handb. Clin. Neurol. 2018, 159, 119-134. [CrossRef]

9. McIsaac, T.L.; Lamberg, E.M.; Muratori, L.M. Building a Framework for a Dual Task Taxonomy. Available online: https://www.hindawi.com/journals/bmri/2015/591475/ (accessed on 14 January 2020).

10. Hillel, I.; Gazit, E.; Nieuwboer, A.; Avanzino, L.; Rochester, L.; Cereatti, A.; Croce, U.D.; Rikkert, M.O.; Bloem, B.R.; Pelosin, E.; et al. Is every-day walking in older adults more analogous to dual-task walking or to usual walking? Elucidating the gaps between gait performance in the lab and during 24/7 monitoring. Eur. Rev. Aging Phys. Act. 2019, 16, 6. [CrossRef]

11. Raffegeau, T.E.; Krehbiel, L.M.; Kang, N.; Thijs, F.J.; Altmann, L.J.P.; Cauraugh, J.H.; Hass, C.J. A meta-analysis: Parkinson's disease and dual-task walking. Parkinsonism Relat. Disord. 2019, 62, 28-35. [CrossRef]

12. Rooney, S.; Ozkul, C.; Paul, L. Correlates of dual-task performance in people with multiple sclerosis: A systematic review. Gait Posture 2020, 81, 172-182. [CrossRef]

13. Yang, L.; Lam, F.M.; Huang, M.; He, C.; Pang, M.Y. Dual-task mobility among individuals with chronic stroke: Changes in cognitive-motor interference patterns and relationship to difficulty level of mobility and cognitive tasks. Eur. J. Phys. Rehabil. Med. 2018, 54, 526-535. [CrossRef] [PubMed]

14. Abdallat, R.; Sharouf, F.; Button, K.; Al-Amri, M. Dual-Task Effects on Performance of Gait and Balance in People with Knee Pain: A Systematic Scoping Review. J. Clin. Med. 2020, 9, 1554. [CrossRef] [PubMed]

15. UzunkulaoĞlu, A.; Kerİm, D.; Ay, S.; Ergİn, S. Effects of single-task versus dual-task training on balance performance in elderly patients with knee osteoarthritis. Arch. Rheumatol. 2020, 35, 35-40. [CrossRef] [PubMed]

16. Bishnoi, A.; Hernandez, M.E. Dual task walking costs in older adults with mild cognitive impairment: A systematic review and meta-analysis. Aging Ment. Health 2020, 1-12. [CrossRef]

17. Montero-Odasso, M.; Muir, S.W.; Speechley, M. Dual-task complexity affects gait in people with mild cognitive impairment: The interplay between gait variability, dual tasking, and risk of falls. Arch. Phys. Med. Rehabil. 2012, 93, 293-299. [CrossRef]

18. Springer, S.; Giladi, N.; Peretz, C.; Yogev, G.; Simon, E.S.; Hausdorff, J.M. Dual-tasking effects on gait variability: The role of aging, falls, and executive function. Mov. Disord. Off. J. Mov. Disord. Soc. 2006, 21, 950-957. [CrossRef]

19. Bayot, M.; Dujardin, K.; Tard, C.; Defebvre, L.; Bonnet, C.T.; Allart, E.; Delval, A. The interaction between cognition and motor control: A theoretical framework for dual-task interference effects on posture, gait initiation, gait and turning. Neurophysiol. Clin. Clin. Neurophysiol. 2018, 48, 361-375. [CrossRef]

20. Kluge, F.; Gaßner, H.; Hannink, J.; Pasluosta, C.; Klucken, J.; Eskofier, B.M. Towards mobile gait analysis: Concurrent validity and test-retest reliability of an inertial measurement system for the assessment of spatio-temporal gait parameters. Sensors 2017, 17, 1522. [CrossRef]

21. Lachin, J.M. The role of measurement reliability in clinical trials. Clin. Trials 2004, 1, 553-566. [CrossRef]

22. Wedege, P.; Steffen, K.; Strøm, V.; Opheim, A.I. Reliability of three-dimensional kinematic gait data in adults with spinal cord injury. J. Rehabil. Assist. Technol. Eng. 2017, 4. [CrossRef]

23. Leszczewska, J.; Czaprowski, D.; Pawłowska, P.; Białobrzewska, K.; Gębicka, A.; Kotwicki, T. Inter-examiner, intra-session and inter-session reliability of gait measurement. Stud. Health Technol. Inform. 2012, 176, 155-158. [PubMed]

24. Girard, O.; Brocherie, F.; Morin, J.-B.; Millet, G.P. Intrasession and intersession reliability of running mechanics during treadmill sprints. Int. J. Sports Physiol. Perform. 2016, 11, 432-439. [CrossRef] [PubMed]

25. Balaguier, R.; Madeleine, P.; Vuillerme, N. Is one trial sufficient to obtain excellent pressure pain threshold reliability in the low back of asymptomatic individuals? a test-retest study. PLoS ONE 2016, 11, e0160866. [CrossRef] [PubMed]

26. Monaghan, K.; Delahunt, E.; Caulfield, B. Increasing the number of gait trial recordings maximises intra-rater reliability of the CODA motion analysis system. Gait Posture 2007, 25, 303-315. [CrossRef] [PubMed]

27. Bloch, M.L.; Jønsson, L.R.; Kristensen, M.T. Introducing a third timed up \& go test trial improves performances of hospitalized and community-dwelling older individuals. J. Geriatr. Phys. Ther. 2001 2017, 40, 121-126. [CrossRef]

28. Fransen, M.; Crosbie, J.; Edmonds, J. Reliability of gait measurements in people with osteoarthritis of the knee. Phys. Ther. 1997, 77, 944-953. [CrossRef] 
29. Haley, S.M.; Fragala-Pinkham, M.A. Interpreting change scores of tests and measures used in physical therapy. Phys. Ther. 2006, 86, 735-743. [CrossRef]

30. Bohn, R.; Cooney, M.; Deodhar, A.; Curtis, J.R.; Golembesky, A. Incidence and prevalence of axial spondyloarthritis: Methodologic challenges and gaps in the literature. Clin. Exp. Rheumatol. 2018, 36, 263-274.

31. Batur, E.B.; Karataş, G.K. Do postural changes affect balance in patients with ankylosing spondylitis? J. Rehabil. Med. 2017, 49,437-440. [CrossRef]

32. Vergara, M.E.; O'Shea, F.D.; Inman, R.D.; Gage, W.H. Postural control is altered in patients with ankylosing spondylitis. Clin. Biomech. 2012, 27, 334-340. [CrossRef]

33. Aydog, E.; Depedibi, R.; Bal, A.; Eksioglu, E.; Unlü, E.; Cakci, A. Dynamic postural balance in ankylosing spondylitis patients. Rheumatology 2006, 45, 445-448. [CrossRef]

34. Bot, S.D.; Caspers, M.; Van Royen, B.J.; Toussaint, H.M.; Kingma, I. Biomechanical analysis of posture in patients with spinal kyphosis due to ankylosing spondylitis: A pilot study. Rheumatology 1999, 38, 441-443. [CrossRef] [PubMed]

35. Koca, T.T.; Göğebakan, H.; Koçyiğit, B.F.; Nacitarhan, V.; Yildir, C.Z. Foot functions in ankylosing spondylitis. Clin. Rheumatol. 2018. [CrossRef] [PubMed]

36. Soulard, J.; Vuillerme, N.; Vaillant, J. Gait characteristics in patients with ankylosing spondylitis: Protocol for a systematic review. JMIR Res. Protoc. 2019, 8, e12470. [CrossRef] [PubMed]

37. Zebouni, L.; Helliwell, P.S.; Howe, A.; Wright, V. Gait analysis in ankylosing spondylitis. Ann. Rheum. Dis. 1992, 51, 898-899. [CrossRef]

38. Mangone, M.; Scettri, P.; Paoloni, M.; Procaccianti, R.; Spadaro, A.; Santilli, V. Pelvis-shoulder coordination during level walking in patients with ankylosing spondylitis. Gait Posture 2011, 34, 1-5. [CrossRef]

39. Del Din, S.; Carraro, E.; Sawacha, Z.; Guiotto, A.; Bonaldo, L.; Masiero, S.; Cobelli, C. Impaired gait in ankylosing spondylitis. Med. Biol. Eng. Comput. 2011, 49, 801-809. [CrossRef]

40. Zhang, G.; Li, J.; Xia, Z.; Xu, W. The gait deviations of ankylosing spondylitis with hip involvement. Clin. Rheumatol. 2019, 38, 1163-1175. [CrossRef]

41. Soulard, J.; Vaillant, J.; Agier, C.-T.; Vuillerme, N. Gait Characteristics in patients with ankylosing spondylitis-A systematic review. J. Clin. Exp. Rheumatol. 2020. in Press. [CrossRef]

42. Soulard, J.; Vuillerme, N.; Gaudin, P.; Grange, L.; Baillet, A.; Cracowski, J.-L.; Juvin, R.; Vaillant, J. Gait as predictor of physical function in axial spondyloarthritis: The prospective longitudinal FOLOMI (Function, Locomotion, Measurement, Inflammation) study protocol. Rheumatol. Int. 2019. [CrossRef]

43. Sieper, J.; Rudwaleit, M.; Baraliakos, X.; Brandt, J.; Braun, J.; Burgos-Vargas, R.; Dougados, M.; Hermann, K.-G.; Landewé, R.; Maksymowych, W.; et al. The Assessment of SpondyloArthritis international Society (ASAS) handbook: A guide to assess spondyloarthritis. Ann. Rheum. Dis. 2009, 68 (Suppl. 2), ii1-ii44. [CrossRef]

44. van der Linden, S.; Valkenburg, H.A.; Cats, A. Evaluation of diagnostic criteria for ankylosing spondylitis. A proposal for modification of the New York criteria. Arthritis Rheum. 1984, 27, 361-368. [CrossRef] [PubMed]

45. Kottner, J.; Audigé, L.; Brorson, S.; Donner, A.; Gajewski, B.J.; Hróbjartsson, A.; Roberts, C.; Shoukri, M.; Streiner, D.L. Guidelines for Reporting Reliability and Agreement Studies (GRRAS) were proposed. J. Clin. Epidemiol. 2011, 64, 96-106. [CrossRef] [PubMed]

46. Wnarifin.Github.io > Sample Size Calculator. Available online: https://wnarifin.github.io/ssc/ssicc.html (accessed on 6 August 2020).

47. Walter, S.D.; Eliasziw, M.; Donner, A. Sample size and optimal designs for reliability studies. Stat. Med. 1998, 17, 101-110. [CrossRef]

48. Song, Y.; Wang, C.; Chen, H. Functional limitation and associated factors in outpatients with ankylosing spondylitis in Southwest China. Clin. Rheumatol. 2017, 36, 871-877. [CrossRef]

49. Graham, J.E.; Ostir, G.V.; Fisher, S.R.; Ottenbacher, K.J. Assessing walking speed in clinical research: A systematic review. J. Eval. Clin. Pract. 2008, 14, 552-562. [CrossRef]

50. Beauchet, O.; Allali, G.; Sekhon, H.; Verghese, J.; Guilain, S.; Steinmetz, J.-P.; Kressig, R.W.; Barden, J.M.; Szturm, T.; Launay, C.P.; et al. Guidelines for assessment of gait and reference values for spatiotemporal gait parameters in older adults: The biomathics and canadian gait consortiums Initiative. Front. Hum. Neurosci. 2017, 11, 353. [CrossRef]

51. Yang, L.; He, C.; Pang, M.Y.C. Reliability and validity of dual-task mobility assessments in people with chronic stroke. PLoS ONE 2016, 11. [CrossRef] 
52. Lefeber, N.; Degelaen, M.; Truyers, C.; Safin, I.; Beckwee, D. Validity and reproducibility of inertial physilog sensors for spatiotemporal gait analysis in patients with stroke. IEEE Trans. Neural Syst. Rehabil. Eng. Publ. IEEE Eng. Med. Biol. Soc. 2019, 27, 1865-1874. [CrossRef]

53. Dadashi, F.; Mariani, B.; Rochat, S.; Büla, C.J.; Santos-Eggimann, B.; Aminian, K. Gait and foot clearance parameters obtained using shoe-worn inertial sensors in a large-population sample of older adults. Sensors 2014, 14, 443-457. [CrossRef]

54. Truong, P.H.; Lee, J.; Kwon, A.-R.; Jeong, G.-M. Stride counting in human walking and walking distance estimation using insole sensors. Sensors 2016, 16, 823. [CrossRef] [PubMed]

55. Anwary, A.R.; Yu, H.; Vassallo, M. An automatic gait feature extraction method for identifying gait asymmetry using wearable sensors. Sensors 2018, 18, 676. [CrossRef]

56. Hollman, J.H.; Childs, K.B.; McNeil, M.L.; Mueller, A.C.; Quilter, C.M.; Youdas, J.W. Number of strides required for reliable measurements of pace, rhythm and variability parameters of gait during normal and dual task walking in older individuals. Gait Posture 2010, 32, 23-28. [CrossRef]

57. Pinzone, O.; Schwartz, M.H.; Baker, R. Comprehensive non-dimensional normalization of gait data. Gait Posture 2016, 44, 68-73. [CrossRef]

58. Hof, A. Scaling gait data to body size. Gait Posture 1996, 4, 222-223. [CrossRef]

59. Błażkiewicz, M.; Wiszomirska, I.; Wit, A. Comparison of four methods of calculating the symmetry of spatial-temporal parameters of gait. Acta Bioeng. Biomech. 2014, 16, $29-35$.

60. Patterson, K.K.; Gage, W.H.; Brooks, D.; Black, S.E.; McIlroy, W.E. Evaluation of gait symmetry after stroke: A comparison of current methods and recommendations for standardization. Gait Posture 2010, 31, 241-246. [CrossRef]

61. Kelly, V.E.; Janke, A.A.; Shumway-Cook, A. Effects of instructed focus and task difficulty on concurrent walking and cognitive task performance in healthy young adults. Exp. Brain Res. Exp. Hirnforsch Exp. Cereb. 2010, 207, 65-73. [CrossRef]

62. Veldkamp, R.; Romberg, A.; Hämäläinen, P.; Giffroy, X.; Moumdjian, L.; Leone, C.; Feys, P.; Baert, I. Test-retest reliability of cognitive-motor interference assessments in walking with various task complexities in persons with multiple sclerosis. Neurorehabil. Neural Repair 2019, 33, 623-634. [CrossRef]

63. Landis, J.R.; Koch, G.G. The measurement of observer agreement for categorical data. Biometrics 1977, 33, 159-174. [CrossRef]

64. Harvill, L.M. Standard error of measurement. Educ. Meas. Issues Pract. 1991, 10, 33-41. [CrossRef]

65. Walton, D.M.; Macdermid, J.C.; Nielson, W.; Teasell, R.W.; Chiasson, M.; Brown, L. Reliability, standard error, and minimum detectable change of clinical pressure pain threshold testing in people with and without acute neck pain. J. Orthop. Sports Phys. Ther. 2011, 41, 644-650. [CrossRef] [PubMed]

66. Venema, D.M.; Hansen, H.; High, R.; Goetsch, T.; Siu, K.-C. Minimal detectable change in dual-task cost for older adults with and without cognitive impairment. J. Geriatr. Phys. Ther. 2001 2018. [CrossRef] [PubMed]

67. Yang, L.; Liao, L.R.; Lam, F.M.H.; He, C.Q.; Pang, M.Y.C. Psychometric properties of dual-task balance assessments for older adults: A systematic review. Maturitas 2015, 80, 359-369. [CrossRef]

68. Yang, L.; Lam, F.M.H.; Liao, L.R.; Huang, M.Z.; He, C.Q.; Pang, M.Y.C. Psychometric properties of dual-task balance and walking assessments for individuals with neurological conditions: A systematic review. Gait Posture 2017, 52, 110-123. [CrossRef]

69. Kobsar, D.; Charlton, J.M.; Tse, C.T.F.; Esculier, J.-F.; Graffos, A.; Krowchuk, N.M.; Thatcher, D.; Hunt, M.A. Validity and reliability of wearable inertial sensors in healthy adult walking: A systematic review and meta-analysis. J. Neuroeng. Rehabil. 2020, 17, 62. [CrossRef]

70. Godfrey, A.; Del Din, S.; Barry, G.; Mathers, J.C.; Rochester, L. Instrumenting gait with an accelerometer: A system and algorithm examination. Med. Eng. Phys. 2015, 37, 400-407. [CrossRef]

71. Del Din, S.; Godfrey, A.; Rochester, L. Validation of an accelerometer to quantify a comprehensive battery of gait characteristics in healthy older adults and parkinson's disease: Toward clinical and at home use. IEEE J. Biomed. Health Inform. 2016, 20, 838-847. [CrossRef]

72. Allet, L.; Armand, S.; de Bie, R.A.; Golay, A.; Monnin, D.; Aminian, K.; de Bruin, E.D. Reliability of diabetic patients' gait parameters in a challenging environment. Gait Posture 2008, 28, 680-686. [CrossRef]

73. Hunter, S.W.; Divine, A.; Frengopoulos, C.; Montero Odasso, M. A framework for secondary cognitive and motor tasks in dual-task gait testing in people with mild cognitive impairment. BMC Geriatr. 2018, 18, 202. [CrossRef] 
74. Saxena, S.; Majnemer, A.; Li, K.; Beauchamp, M.; Gagnon, I. A cross-sectional analysis on the effects of age on dual tasking in typically developing children. Psychol. Res. 2019, 83, 104-115. [CrossRef] [PubMed]

75. Chen, A.; Kirkland, M.C.; Wadden, K.P.; Wallack, E.M.; Ploughman, M. Reliability of gait and dual-task measures in multiple sclerosis. Gait Posture 2020, 78, 19-25. [CrossRef]

76. Decavel, P.; Moulin, T.; Sagawa, Y. Gait tests in multiple sclerosis: Reliability and cut-off values. Gait Posture 2019, 67, 37-42. [CrossRef] [PubMed]

77. Muhaidat, J.; Kerr, A.; Evans, J.J.; Skelton, D.A. The test-retest reliability of gait-related dual task performance in community-dwelling fallers and non-fallers. Gait Posture 2013, 38, 43-50. [CrossRef] [PubMed]

78. Plummer, P.; Grewal, G.; Najafi, B.; Ballard, A. Instructions and skill level influence reliability of dual-task performance in young adults. Gait Posture 2015, 41, 964-967. [CrossRef]

79. Brown, K.C.; Hanson, H.M.; Firmani, F.; Liu, D.; McAllister, M.M.; Merali, K.; Puyat, J.H.; Ashe, M.C. Gait speed and variability for usual pace and pedestrian crossing conditions in older adults using the gaitrite walkway. Gerontol. Geriatr. Med. 2015. [CrossRef]

80. Afilalo, J.; Sharma, A.; Zhang, S.; Brennan, J.M.; Edwards, F.H.; Mack, M.J.; McClurken, J.B.; Cleveland, J.C.; Smith, P.K.; Shahian, D.M.; et al. Gait speed and 1-year mortality following cardiac surgery: A landmark analysis from the society of thoracic surgeons adult cardiac surgery database. J. Am. Heart Assoc. 2018, 7, e010139. [CrossRef]

81. Brach, J.S.; Perera, S.; Studenski, S.; Newman, A.B. The reliability and validity of measures of gait variability in community-dwelling older adults. Arch. Phys. Med. Rehabil. 2008, 89, 2293-2296. [CrossRef]

82. Stone, M.A.; Pomeroy, E.; Keat, A.; Sengupta, R.; Hickey, S.; Dieppe, P.; Gooberman-Hill, R.; Mogg, R.; Richardson, J.; Inman, R.D. Assessment of the impact of flares in ankylosing spondylitis disease activity using the Flare Illustration. Rheumatology 2008, 47, 1213-1218. [CrossRef]

83. Najafi, S.; Rezasoltani, Z.; Abedi, M. Effects of mechanical low back pain in spatiotemporal parameters of gait. J. Arch. Mil. Med. 2018, 6. [CrossRef]

84. Smith, E.; Cusack, T.; Cunningham, C.; Blake, C. The influence of a cognitive dual task on the gait parameters of healthy older adults: A systematic review and meta-analysis. J. Aging Phys. Act. 2017, 25, 671-686. [CrossRef] [PubMed]

85. Çınar, E.; Akkoç, Y.; Karapolat, H.; Durusoy, R.; Keser, G. Postural deformities: Potential morbidities to cause balance problems in patients with ankylosing spondylitis? Eur. J. Rheumatol. 2016, 3, 5-9. [CrossRef] [PubMed]

Publisher's Note: MDPI stays neutral with regard to jurisdictional claims in published maps and institutional affiliations.

(C) 2020 by the authors. Licensee MDPI, Basel, Switzerland. This article is an open access article distributed under the terms and conditions of the Creative Commons Attribution (CC BY) license (http://creativecommons.org/licenses/by/4.0/). 